\title{
Fourier transform of a Gaussian measure on the Heisenberg group
}

\author{
Mátyás Barczy, Gyula Pap* \\ Faculty of Informatics, University of Debrecen, Pf. 12, 4010 Debrecen, Hungary
}

Received 5 July 2004; received in revised form 8 July 2005; accepted 12 July 2005

Available online 7 December 2005

\begin{abstract}
An explicit formula is derived for the Fourier transform of a Gaussian measure on the Heisenberg group at the Schrödinger representation. Using this explicit formula, necessary and sufficient conditions are given for the convolution of two Gaussian measures to be a Gaussian measure.
\end{abstract}

(c) 2005 Elsevier SAS. All rights reserved.

\section{Résumé}

Une formule explicite est donnée pour la transformée de Fourier de la mesure gaussienne sur le groupe d'Heisenberg dans la représentation de Schrödinger. A l'aide de celle-ci, des conditions nécessaires et suffisantes sont données pour que la convolée de deux mesures gaussiennes soit gaussienne.

(c) 2005 Elsevier SAS. All rights reserved.

MSC: 60B15

Keywords: Heisenberg group; Fourier transforms; Gaussian measures

\section{Introduction}

Fourier transforms of probability measures on a locally compact topological group play an important role in several problems concerning convolution and weak convergence of probability measures. Indeed, the Fourier transform of the convolution of two probability measures is the product of their Fourier transforms, and in case of many groups the continuity theorem holds, namely, weak convergence of probability measures is equivalent to the pointwise convergence of their Fourier transforms. Moreover, the Fourier transform is injective, i.e., if the Fourier transforms of two probability measures coincide at each point then the measures coincide. (See the properties of the Fourier transform, e.g., in Heyer [7, Chapter I].) In case of a locally compact Abelian group, an explicit formula is available for the Fourier transform of an arbitrary infinitely divisible probability measure (see Parthatsarathy [11]). The case of non-Abelian groups is much more complicated. For Lie groups, Tomé [16] proposed a method how to calculate Fourier transforms based on Feynman's path integral and discussed the physical motivation, but explicit expressions have been derived only in very special cases.

\footnotetext{
* Corresponding author.

E-mail addresses: barczy@inf.unideb.hu (M. Barczy), papgy@inf.unideb.hu (G. Pap).
} 
In this paper Gaussian measures will be investigated on the 3-dimensional Heisenberg group $\mathbb{H}$ which can be obtained by furnishing $\mathbb{R}^{3}$ with its natural topology and with the product

$$
\left(g_{1}, g_{2}, g_{3}\right)\left(h_{1}, h_{2}, h_{3}\right)=\left(g_{1}+h_{1}, g_{2}+h_{2}, g_{3}+h_{3}+\frac{1}{2}\left(g_{1} h_{2}-g_{2} h_{1}\right)\right) .
$$

The Schrödinger representations $\left\{\pi_{ \pm \lambda}: \lambda>0\right\}$ of $\mathbb{H}$ are representations in the group of unitary operators of the complex Hilbert space $L^{2}(\mathbb{R})$ given by

$$
\left[\pi_{ \pm \lambda}(g) u\right](x):=\mathrm{e}^{ \pm \mathrm{i}\left(\lambda g_{3}+\sqrt{\lambda} g_{2} x+\lambda g_{1} g_{2} / 2\right)} u\left(x+\sqrt{\lambda} g_{1}\right)
$$

for $g=\left(g_{1}, g_{2}, g_{3}\right) \in \mathbb{H}, u \in L^{2}(\mathbb{R})$ and $x \in \mathbb{R}$. The value of the Fourier transform of a probability measure $\mu$ on $\mathbb{H}$ at the Schrödinger representation $\pi_{ \pm \lambda}$ is the bounded linear operator $\hat{\mu}\left(\pi_{ \pm \lambda}\right): L^{2}(\mathbb{R}) \rightarrow L^{2}(\mathbb{R})$ given by

$$
\hat{\mu}\left(\pi_{ \pm \lambda}\right) u:=\int_{\mathbb{H}} \pi_{ \pm \lambda}(g) u \mu(\mathrm{d} g), \quad u \in L^{2}(\mathbb{R}),
$$

interpreted as a Bochner integral.

Let $\left(\mu_{t}\right)_{t \geqslant 0}$ be a Gaussian convolution semigroup of probability measures on $\mathbb{H}$ (see Section 2). By a result of Siebert [13, Proposition 3.1, Lemma 3.1], $\left(\widehat{\mu}_{t}\left(\pi_{ \pm \lambda}\right)\right)_{t \geqslant 0}$ is a strongly continuous semigroup of contractions on $L^{2}(\mathbb{R})$ with infinitesimal generator

$$
N\left(\pi_{ \pm \lambda}\right)=\alpha_{1} I+\alpha_{2} x+\alpha_{3} D+\alpha_{4} x^{2}+\alpha_{5}(x D+D x)+\alpha_{6} D^{2},
$$

where $\alpha_{1}, \ldots, \alpha_{6}$ are certain complex numbers (depending on $\left(\mu_{t}\right)_{t} \geqslant 0$, see Remark 3.1), $I$ denotes the identity operator on $L^{2}(\mathbb{R}), x$ is the multiplication by the variable $x$, and $D u(x)=u^{\prime}(x)$. One of our purposes is to determine the action of the operators

$$
\widehat{\mu_{t}}\left(\pi_{ \pm \lambda}\right)=\mathrm{e}^{t N\left(\pi_{ \pm \lambda}\right)}, \quad t \geqslant 0
$$

on $L^{2}(\mathbb{R})$. (Here the notation $\left(\mathrm{e}^{t A}\right)_{t \geqslant 0}$ means a semigroup of operators with infinitesimal generator $A$.) When $N\left(\pi_{ \pm \lambda}\right)$ has the special form $\frac{1}{2}\left(D^{2}-x^{2}\right)$, the celebrated Mehler's formula gives us

$$
\mathrm{e}^{t\left(D^{2}-x^{2}\right) / 2} u(x)=\frac{1}{\sqrt{2 \pi \sinh t}} \int_{\mathbb{R}} \exp \left\{-\frac{\left(x^{2}+y^{2}\right) \cosh t-2 x y}{2 \sinh t}\right\} u(y) \mathrm{d} y
$$

for all $t>0, u \in L^{2}(\mathbb{R})$ and $x \in \mathbb{R}$, see, e.g., Taylor [15], Davies [4]. Our Theorem 3.1 in Section 3 can be regarded as a generalization of Mehler's formula.

It turns out that $\widehat{\mu_{t}}\left(\pi_{ \pm \lambda}\right)=\mathrm{e}^{t N\left(\pi_{ \pm \lambda}\right)}, t \geqslant 0$ are again integral operators on $L^{2}(\mathbb{R})$ if $\alpha_{6}$ is a positive real number. One of the main results of the present paper is an explicit formula for the kernel function of these integral operators (see Theorem 3.1). We apply a probabilistic method using that the Fourier transform $\hat{\mu}\left(\pi_{ \pm \lambda}\right)$ of an absolutely continuous probability measure $\mu$ on $\mathbb{H}$ can be derived from the Euclidean Fourier transform of $\mu$ considering $\mu$ as a measure on $\mathbb{R}^{3}$ (see Proposition 4.1). We note that a random walk approach might provide a different proof of Theorem 3.1, but we think that it would not be simpler than ours.

The second part of the paper deals with convolutions of Gaussian measures on $\mathbb{H}$. The convolution of two Gaussian measures on a locally compact Abelian group is again a Gaussian measure (it can be proved by the help of Fourier transforms; see Parthatsarathy [11]). We prove that a convolution of Gaussian measures on $\mathbb{H}$ is almost never a Gaussian measure. More exactly, we obtain the following result (using our explicit formula for the Fourier transforms).

Theorem 1.1. Let $\mu^{\prime}$ and $\mu^{\prime \prime}$ be Gaussian measures on $\mathbb{H}$. Then the convolution $\mu^{\prime} * \mu^{\prime \prime}$ is a Gaussian measure on $\mathbb{H}$ if and only if one of the following conditions holds:

(C1) there exist elements $Y_{0}^{\prime}, Y_{0}^{\prime \prime}, Y_{1}, Y_{2}$ in the Lie algebra of $\mathbb{H}$ such that $\left[Y_{1}, Y_{2}\right]=0$, the support of $\mu^{\prime}$ is contained in $\exp \left\{Y_{0}^{\prime}+\mathbb{R} \cdot Y_{1}+\mathbb{R} \cdot Y_{2}\right\}$ and the support of $\mu^{\prime \prime}$ is contained in $\exp \left\{Y_{0}^{\prime \prime}+\mathbb{R} \cdot Y_{1}+\mathbb{R} \cdot Y_{2}\right\}$. (Equivalently, there exists an Abelian subgroup $\mathbb{G}$ of $\mathbb{H}$ such that $\operatorname{supp}\left(\mu^{\prime}\right)$ and $\operatorname{supp}\left(\mu^{\prime \prime}\right)$ are contained in "Euclidean cosets" of $\mathbb{G}$.) 
(C2) there exist a Gaussian semigroup $\left(\mu_{t}\right)_{t \geqslant 0}$ and $t^{\prime}, t^{\prime \prime} \geqslant 0$ and a Gaussian measure $v$ such that $\operatorname{supp}(v)$ is contained in the center of $\mathbb{H}$ and either $\mu^{\prime}=\mu_{t^{\prime}}, \mu^{\prime \prime}=\mu_{t^{\prime \prime}} * v$ or $\mu^{\prime}=\mu_{t^{\prime}} * v, \mu^{\prime \prime}=\mu_{t^{\prime \prime}}$ holds. (Equivalently, $\mu^{\prime}$ and $\mu^{\prime \prime}$ are sitting on the same Gaussian semigroup modulo a Gaussian measure with support contained in the center of $\mathbb{H}$.)

We note that in case of $(\mathrm{C} 1), \mu^{\prime}$ and $\mu^{\prime \prime}$ are Gaussian measures also in the "Euclidean sense" (i.e., considering them as measures on $\mathbb{R}^{3}$ ). Moreover, Theorem 6.1 contains an explicit formula for the Fourier transform of a convolution of arbitrary Gaussian measures on $\mathbb{H}$.

The structure of the present work is similar to Pap [10]. Theorems 1.1 and 3.1 of the present paper are generalizations of the corresponding results for symmetric Gaussian measures on $\mathbb{H}$ due to Pap [10]. We summarize briefly the new ingredients needed in the present paper. Comparing Lemma 6.1 in Pap [10] and Proposition 5.1 of the present paper, one can realize that now we have to calculate a much more complicated (Euclidean) Fourier transform (see (5.5)). For this reason we generalized a result due to Chaleyat-Maurel [3] (see Lemma 5.2). We note that using Lemma 6.2 one can easily derive Theorem 1.1 in Pap [10] from Theorem 1.1 of the present paper.

It is natural to ask whether we can prove our results for non-symmetric Gaussian measures using only the results for symmetric Gaussian measures. The answer is no. The reason for this is that in case of $\mathbb{H}$ the convolution of a symmetric Gaussian measure and a Dirac measure is in general not a Gaussian measure. For example, if $a=(1,0,0) \in \mathbb{H}$ and $\left(\mu_{t}\right)_{t \geqslant 0}$ is a Gaussian semigroup with infinitesimal generator $X_{1}^{2}+X_{2}^{2}$, then using Lemma 4.2, one can easily check that $\mu_{1} * \varepsilon_{a}$ is not a Gaussian measure on $\mathbb{H}$, where $\varepsilon_{a}$ denotes the Dirac measure concentrated on the element $a \in \mathbb{H}$. (For the definition of an infinitesimal generator and $X_{1}, X_{2}, X_{3}$, see Section 2.)

We note that if the convolution of two Gaussian measures on $\mathbb{H}$ is again a Gaussian measure on $\mathbb{H}$, then the corresponding infinitesimal generators not necessarily commute, nor even if the infinitesimal generator corresponding to the convolution is the sum of the original infinitesimal generators. Now we give an illuminating counterexample. Let $\mu^{\prime}$ and $\mu^{\prime \prime}$ be Gaussian measures on $\mathbb{H}$ such that the corresponding Gaussian semigroups have infinitesimal generators

$$
N^{\prime}=\frac{1}{2}\left(X_{1}+X_{2}\right)^{2} \quad \text { and } \quad N^{\prime \prime}=\frac{1}{2}\left(X_{1}+X_{2}\right)^{2}+X_{1} X_{3}, \quad \text { respectively. }
$$

Using Theorem 6.2 and Lemma 6.2, $\mu^{\prime} * \mu^{\prime \prime}$ is a symmetric Gaussian measure on $\mathbb{H}$ such that the corresponding Gaussian semigroup has infinitesimal generator $N^{\prime}+N^{\prime \prime}$. But $N^{\prime}$ and $N^{\prime \prime}$ do not commute. Indeed, $N^{\prime} N^{\prime \prime}-N^{\prime \prime} N^{\prime}=$ $-\left(X_{1}+X_{2}\right) X_{3}^{2} \neq 0$.

At the end of our paper we formulate Theorem 1.1 in the important special case of centered Gaussian measures for which the corresponding Gaussian semigroups are stable in the sense of Hazod. This kind of Gaussian measures arises in a standard version of central limit theorems on $\mathbb{H}$ proved by Wehn [17]. In this special case Theorem $1.1 \mathrm{can}$ be derived from the results for symmetric Gaussian measures in Pap [10].

\section{Preliminaries}

The Heisenberg group $\mathbb{H}$ is a Lie group with Lie algebra $\mathcal{H}$, which can be realized as the vector space $\mathbb{R}^{3}$ furnished with multiplication

$$
\left[\left(p_{1}, p_{2}, p_{3}\right),\left(q_{1}, q_{2}, q_{3}\right)\right]=\left(0,0, p_{1} q_{2}-p_{2} q_{1}\right) .
$$

An element $X \in \mathcal{H}$ can be regarded as a left-invariant differential operator on $\mathbb{H}$, namely, for continuously differentiable functions $f: \mathbb{H} \rightarrow \mathbb{R}$ we put

$$
X f(g):=\lim _{t \rightarrow 0} t^{-1}(f(g \exp (t X))-f(g)), \quad g \in \mathbb{H},
$$

where the exponential mapping exp: $\mathcal{H} \rightarrow \mathbb{H}$ is now the identity mapping.

A family $\left(\mu_{t}\right)_{t} \geqslant 0$ of probability measures on $\mathbb{H}$ is said to be a (continuous) convolution semigroup if we have $\mu_{s} * \mu_{t}=\mu_{s+t}$ for all $s, t \geqslant 0$, and $\lim _{t \downarrow 0} \mu_{t}=\mu_{0}=\varepsilon_{e}$, where $e=(0,0,0)$ is the unit element of $\mathbb{H}$. Its infinitesimal generator is defined by

$$
(N f)(g):=\lim _{t \downarrow 0} t^{-1} \int_{\mathbb{H}}(f(g h)-f(g)) \mu_{t}(\mathrm{~d} h), \quad g \in \mathbb{H},
$$


for suitable functions $f: \mathbb{H} \rightarrow \mathbb{R}$. (The infinitesimal generator is always defined for infinitely differentiable functions $f: \mathbb{H} \rightarrow \mathbb{R}$ with compact support.) A convolution semigroup $\left(\mu_{t}\right)_{t \geqslant 0}$ is called a Gaussian semigroup if $\lim _{t \downarrow 0} t^{-1} \mu_{t}(\mathbb{H} \backslash U)=0$ for all (Borel) neighbourhoods $U$ of $e$. Let $\left\{X_{1}, X_{2}, X_{3}\right\}$ denote the natural basis in $\mathcal{H}$ (that is, $\exp X_{1}=(1,0,0), \exp X_{2}=(0,1,0)$ and $\left.\exp X_{3}=(0,0,1)\right)$. It is known that a convolution semigroup $\left(\mu_{t}\right)_{t \geqslant 0}$ is a Gaussian semigroup if and only if its infinitesimal generator has the form

$$
N=\sum_{k=1}^{3} a_{k} X_{k}+\frac{1}{2} \sum_{j=1}^{3} \sum_{k=1}^{3} b_{j, k} X_{j} X_{k},
$$

where $a=\left(a_{1}, a_{2}, a_{3}\right) \in \mathbb{R}^{3}$ and $B=\left(b_{j, k}\right)_{1 \leqslant j, k \leqslant 3}$ is a real, symmetric, positive semidefinite matrix. A probability measure $\mu$ on $\mathbb{H}$ is called a Gaussian measure if there exists a Gaussian semigroup $\left(\mu_{t}\right)_{t \geqslant 0}$ such that $\mu=\mu_{1}$. A Gaussian measure on $\mathbb{H}$ can be embedded only in a uniquely determined Gaussian semigroup (see Baldi [2], Pap [9]). (Neuenschwander [8] showed that a Gaussian measure on $\mathbb{H}$ can not be embedded in a non-Gaussian convolution semigroup.) Thus for a vector $a=\left(a_{1}, a_{2}, a_{3}\right) \in \mathbb{R}^{3}$ and a real, symmetric, positive semidefinite matrix $B=\left(b_{j, k}\right)_{1 \leqslant j, k \leqslant 3}$ we can speak about the Gaussian measure $\mu$ with parameters $(a, B)$ which is by definition $\mu:=\mu_{1}$, where $\left(\mu_{t}\right)_{t \geqslant 0}$ is the Gaussian semigroup with infinitesimal generator $N$ given by (2.1). If $v$ is a Gaussian measure with parameters $(a, B)$ and $\left(v_{s}\right)_{s} \geqslant 0$ is the Gaussian semigroup with infinitesimal generator $N$ given by (2.1) then $v_{t}$ is a Gaussian measure with parameters $(t a, t B)$ for all $t \geqslant 0$, since $\mu_{s}:=v_{s t}, s \geqslant 0$ defines a Gaussian semigroup with infinitesimal generator $t N$. Hence $v_{t}=\mu_{1}$, so it will be sufficient to calculate the Fourier transform of $\mu_{1}$.

Let us consider a Gaussian semigroup $\left(\mu_{t}\right)_{t} \geqslant 0$ with parameters $(a, B)$ on $\mathbb{H}$. Its infinitesimal generator $N$ can be also written in the form

$$
N=Y_{0}+\frac{1}{2} \sum_{j=1}^{d} Y_{j}^{2},
$$

where $0 \leqslant d \leqslant 3$ and

$$
Y_{0}=\sum_{k=1}^{3} a_{k} X_{k}, \quad Y_{j}=\sum_{k=1}^{3} \sigma_{k, j} X_{k}, \quad 1 \leqslant j \leqslant d,
$$

where $\Sigma=\left(\sigma_{k, j}\right)$ is a $3 \times d$ matrix with $\operatorname{rank}(\Sigma)=\operatorname{rank}(B)=d$. Moreover, $B=\Sigma \cdot \Sigma^{\top}$. Then the measure $\mu_{t}$ can be described as the distribution of the random vector $Z(t)=\left(Z_{1}(t), Z_{2}(t), Z_{3}(t)\right)$ with values in $\mathbb{R}^{3}$, where

$$
\begin{aligned}
Z_{1}(t) & =a_{1} t+\sum_{k=1}^{d} \sigma_{1, k} W_{k}(t), \quad Z_{2}(t)=a_{2} t+\sum_{k=1}^{d} \sigma_{2, k} W_{k}(t), \\
Z_{3}(t) & =a_{3} t+\sum_{k=1}^{d} \sigma_{3, k} W_{k}(t)+\frac{1}{2} \int_{0}^{t}\left(Z_{1}(s) \mathrm{d} Z_{2}(s)-Z_{2}(s) \mathrm{d} Z_{1}(s)\right) \\
& =a_{3} t+\sum_{k=1}^{d} \sigma_{3, k} W_{k}(t)+\sum_{k=1}^{d}\left(a_{2} \sigma_{1, k}-a_{1} \sigma_{2, k}\right) W_{k}^{*}(t)+\sum_{1 \leqslant k<\ell \leqslant d}\left(\sigma_{1, k} \sigma_{2, \ell}-\sigma_{1, \ell} \sigma_{2, k}\right) W_{k, \ell}(t),
\end{aligned}
$$

where $\left(W_{1}(t), \ldots, W_{d}(t)\right)_{t \geqslant 0}$ is a standard Wiener process in $\mathbb{R}^{d}$ and

$$
\begin{aligned}
W_{k}^{*}(t) & :=\frac{1}{2}\left(\int_{0}^{t} W_{k}(s) \mathrm{d} s-\int_{0}^{t} s \mathrm{~d} W_{k}(s)\right), \\
W_{k, \ell}(t) & :=\frac{1}{2}\left(\int_{0}^{t} W_{k}(s) \mathrm{d} W_{\ell}(s)-\int_{0}^{t} W_{\ell}(s) \mathrm{d} W_{k}(s)\right) .
\end{aligned}
$$

(See, e.g., Roynette [12].) The process $\left(W_{k, \ell}(t)\right)_{t \geqslant 0}$ is the so-called Lévy's stochastic area swept by the process $\left(W_{k}(s), W_{\ell}(s)\right)_{s \in[0, t]}$ on $\mathbb{R}^{2}$. 


\section{Fourier transform of a Gaussian measure}

The Schrödinger representations are infinite dimensional, irreducible, unitary representations, and each irreducible, unitary representation is unitarily equivalent with one of the Schrödinger representations or with $\chi_{\alpha, \beta}$ for some $\alpha, \beta \in \mathbb{R}$, where $\chi_{\alpha, \beta}$ is a one-dimensional representation given by

$$
\chi_{\alpha, \beta}(g):=\mathrm{e}^{\mathrm{i}\left(\alpha g_{1}+\beta g_{2}\right)}, \quad g=\left(g_{1}, g_{2}, g_{3}\right) \in \mathbb{H} .
$$

The value of the Fourier transform of a probability measure $\mu$ on $\mathbb{H}$ at the representation $\chi_{\alpha, \beta}$ is

$$
\hat{\mu}\left(\chi_{\alpha, \beta}\right):=\int_{\mathbb{H}} \chi_{\alpha, \beta}(g) \mu(\mathrm{d} g)=\int_{\mathbb{H}} \mathrm{e}^{\mathrm{i}\left(\alpha g_{1}+\beta g_{2}\right)} \mu(\mathrm{d} g)=\tilde{\mu}(\alpha, \beta, 0),
$$

where $\tilde{\mu}: \mathbb{R}^{3} \rightarrow \mathbb{C}$ denotes the Euclidean Fourier transform of $\mu$,

$$
\tilde{\mu}(\alpha, \beta, \gamma):=\int_{\mathbb{H}} \mathrm{e}^{\mathrm{i}\left(\alpha g_{1}+\beta g_{2}+\gamma g_{3}\right)} \mu(\mathrm{d} g) .
$$

Let us consider a Gaussian semigroup $\left(\mu_{t}\right)_{t \geqslant 0}$ with parameters $(a, B)$ on $\mathbb{H}$. The Fourier transform of $\mu:=\mu_{1}$ at the one-dimensional representations can be calculated easily, since the description of $\left(\mu_{t}\right)_{t \geqslant 0}$ given in Section 2 implies that

$$
\hat{\mu}\left(\chi_{\alpha, \beta}\right)=\mathrm{E} \exp \left\{\mathrm{i}\left(\alpha a_{1}+\beta a_{2}\right)+\mathrm{i}\left(\alpha \sum_{k=1}^{d} \sigma_{1, k} W_{k}(1)+\beta \sum_{k=1}^{d} \sigma_{2, k} W_{k}(1)\right)\right\}
$$

for $\alpha, \beta \in \mathbb{R}$. The random variable

$$
\left(\sum_{k=1}^{d} \sigma_{1, k} W_{k}(1), \sum_{k=1}^{d} \sigma_{2, k} W_{k}(1)\right)
$$

has a normal distribution with zero mean and covariance matrix

$$
\left[\begin{array}{lll}
\sigma_{1,1} & \ldots & \sigma_{1, d} \\
\sigma_{2,1} & \ldots & \sigma_{2, d}
\end{array}\right]\left[\begin{array}{cc}
\sigma_{1,1} & \sigma_{2,1} \\
\vdots & \vdots \\
\sigma_{1, d} & \sigma_{2, d}
\end{array}\right]=\left[\begin{array}{ll}
b_{1,1} & b_{1,2} \\
b_{2,1} & b_{2,2}
\end{array}\right]
$$

since $\Sigma \Sigma^{\top}=B$. Consequently,

$$
\hat{\mu}\left(\chi_{\alpha, \beta}\right)=\exp \left\{\mathrm{i}\left(\alpha a_{1}+\beta a_{2}\right)-\frac{1}{2}\left(b_{1,1} \alpha^{2}+2 b_{1,2} \alpha \beta+b_{2,2} \beta^{2}\right)\right\} .
$$

One of the main results of the present paper is an explicit formula for the Fourier transform of a Gaussian measure on the Heisenberg group $\mathbb{H}$ at the Schrödinger representations.

Theorem 3.1. Let $\mu$ be a Gaussian measure on $\mathbb{H}$ with parameters $(a, B)$. Then

$$
\left[\hat{\mu}\left(\pi_{ \pm \lambda}\right) u\right](x)= \begin{cases}\int_{\mathbb{R}} K_{ \pm \lambda}(x, y) u(y) \mathrm{d} y & \text { if } b_{1,1}>0, \\ L_{ \pm \lambda}(x) u\left(x+\sqrt{\lambda} a_{1}\right) & \text { if } b_{1,1}=0,\end{cases}
$$

for $u \in L^{2}(\mathbb{R}), x \in \mathbb{R}$, where

$$
K_{ \pm \lambda}(x, y):=C_{ \pm \lambda}(B) \exp \left\{-\frac{1}{2} \mathbf{z}^{\top} D_{ \pm \lambda}(a, B) \mathbf{z}\right\}, \quad \mathbf{z}:=(x, y, 1)^{\top},
$$


where, with $\delta:=\sqrt{b_{1,1} b_{2,2}-b_{1,2}^{2}}, \delta_{1}:=b_{1,1} b_{2,3}-b_{1,2} b_{1,3}, \delta_{2}:=a_{1} b_{1,2}-a_{2} b_{1,1}$,

$$
C_{ \pm \lambda}(B):= \begin{cases}\frac{1}{\sqrt{2 \pi \lambda b_{1,1}}} & \text { if } \delta=0, \\ \sqrt{\frac{\delta}{2 \pi b_{1,1} \sinh (\lambda \delta)}} & \text { if } \delta>0,\end{cases}
$$

and $D_{ \pm \lambda}(a, B)=\left(d_{j, k}^{ \pm \lambda}(a, B)\right)_{1 \leqslant j, k \leqslant 3}$ are symmetric matrices defined for $b_{1,1}>0$ and $\delta=0$ by

$$
\begin{aligned}
& d_{1,1}^{ \pm \lambda}(a, B):=\frac{\lambda^{-1} \pm \mathrm{i} b_{1,2}}{b_{1,1}}, \quad d_{1,2}^{ \pm \lambda}(a, B):=-\frac{1}{\lambda b_{1,1}}, \quad d_{2,2}^{ \pm \lambda}(a, B):=\frac{\lambda^{-1} \mp \mathrm{i} b_{1,2}}{b_{1,1}}, \\
& d_{1,3}^{ \pm \lambda}(a, B):=\frac{a_{1} \pm \mathrm{i} \lambda b_{1,3}}{\sqrt{\lambda} b_{1,1}} \pm \mathrm{i} \frac{\sqrt{\lambda} \delta_{2}}{2 b_{1,1}}, \quad d_{2,3}^{ \pm \lambda}(a, B):=-\frac{a_{1} \pm \mathrm{i} \lambda b_{1,3}}{\sqrt{\lambda} b_{1,1}} \pm \mathrm{i} \frac{\sqrt{\lambda} \delta_{2}}{2 b_{1,1}}, \\
& d_{3,3}^{ \pm \lambda}(a, B):=\frac{\left(a_{1} \pm \mathrm{i} \lambda b_{1,3}\right)^{2}}{b_{1,1}}+\frac{\lambda^{2} \delta_{2}^{2}}{12 b_{1,1}}+\lambda^{2} b_{3,3} \mp 2 \mathrm{i} \lambda a_{3},
\end{aligned}
$$

and for $\delta>0$ by

$$
\begin{aligned}
& d_{1,1}^{ \pm \lambda}(a, B):=\frac{\delta \operatorname{coth}(\lambda \delta) \pm \mathrm{i} b_{1,2}}{b_{1,1}}, \quad d_{1,2}^{ \pm \lambda}(a, B):=-\frac{\delta}{b_{1,1} \sinh (\lambda \delta)}, \quad d_{2,2}^{ \pm \lambda}(a, B):=\frac{\delta \operatorname{coth}(\lambda \delta) \mp \mathrm{i} b_{1,2}}{b_{1,1}}, \\
& d_{1,3}^{ \pm \lambda}(a, B):=\frac{a_{1} \pm \mathrm{i} \lambda b_{1,3}}{\sqrt{\lambda} b_{1,1}}+\frac{\lambda \delta_{1} \pm \mathrm{i} \delta_{2}}{\sqrt{\lambda} b_{1,1} \delta \operatorname{coth}(\lambda \delta / 2)}, \quad d_{2,3}^{ \pm \lambda}(a, B):=-\frac{a_{1} \pm \mathrm{i} \lambda b_{1,3}}{\sqrt{\lambda} b_{1,1}}+\frac{\lambda \delta_{1} \pm \mathrm{i} \delta_{2}}{\sqrt{\lambda} b_{1,1} \delta \operatorname{coth}(\lambda \delta / 2)}, \\
& d_{3,3}^{ \pm \lambda}(a, B):=\frac{\left(a_{1} \pm \mathrm{i} \lambda b_{1,3}\right)^{2}}{b_{1,1}}-\frac{\left(\lambda \delta_{1} \pm \mathrm{i} \delta_{2}\right)^{2}}{\lambda b_{1,1} \delta^{3}}(\lambda \delta-2 \tanh (\lambda \delta / 2))+\lambda^{2} b_{3,3} \mp 2 \mathrm{i} \lambda a_{3},
\end{aligned}
$$

and

$$
\begin{aligned}
L_{ \pm \lambda}(x):=\exp \{ & \pm \frac{\mathrm{i} \sqrt{\lambda}}{2}\left(\sqrt{\lambda}\left(2 a_{3}+a_{1} a_{2}\right)+2 a_{2} x\right)-\frac{\lambda^{2}}{6}\left(3 b_{3,3}+3 a_{1} b_{2,3}+a_{1}^{2} b_{2,2}\right) \\
& \left.-\frac{\lambda^{3 / 2}}{2}\left(2 b_{2,3}+a_{1} b_{2,2}\right) x-\frac{\lambda}{2} b_{2,2} x^{2}\right\} .
\end{aligned}
$$

We prove this theorem in Section 5.

Remark 3.1. Consider a Gaussian convolution semigroup $\left(\mu_{t}\right)_{t \geqslant 0}$ with infinitesimal generator $N$ given in (2.1). Siebert [13, Proposition 3.1, Lemma 3.1] proved that $\left(\widehat{\mu_{t}}\left(\pi_{ \pm \lambda}\right)\right)_{t \geqslant 0}$ is a strongly continuous semigroup of contractions on $L^{2}(\mathbb{R})$ with infinitesimal generator

$$
N\left(\pi_{ \pm \lambda}\right)=\sum_{k=1}^{3} a_{k} X_{k}\left(\pi_{ \pm \lambda}\right)+\frac{1}{2} \sum_{j=1}^{3} \sum_{k=1}^{3} b_{j, k} X_{j}\left(\pi_{ \pm \lambda}\right) X_{k}\left(\pi_{ \pm \lambda}\right),
$$

where

$$
X\left(\pi_{ \pm \lambda}\right) u:=\lim _{t \rightarrow 0} t^{-1}\left(\pi_{ \pm \lambda}(\exp (t X)) u-u\right)
$$

for all differentiable vectors $u$. Hence

$$
\begin{aligned}
& {\left[X_{1}\left(\pi_{ \pm \lambda}\right) u\right](x)=\sqrt{\lambda} u^{\prime}(x)=\sqrt{\lambda} D u(x),} \\
& {\left[X_{2}\left(\pi_{ \pm \lambda}\right) u\right](x)= \pm \mathrm{i} \sqrt{\lambda} x u(x),} \\
& {\left[X_{3}\left(\pi_{ \pm \lambda}\right) u\right](x)= \pm \mathrm{i} \lambda u(x)}
\end{aligned}
$$


for all $x \in \mathbb{R}$. Consequently,

$$
N\left(\pi_{ \pm \lambda}\right)=\alpha_{1} I+\alpha_{2} x+\alpha_{3} D+\alpha_{4} x^{2}+\alpha_{5}(x D+D x)+\alpha_{6} D^{2},
$$

where

$$
\begin{aligned}
& \alpha_{1}=-\frac{1}{2} \lambda^{2} b_{3,3} \pm \mathrm{i} \lambda a_{3}, \quad \alpha_{2}=-\lambda^{3 / 2} b_{2,3} \pm \mathrm{i} \lambda^{1 / 2} a_{2}, \quad \alpha_{3}=\lambda^{1 / 2} a_{1} \pm \mathrm{i} \lambda^{3 / 2} b_{1,3}, \\
& \alpha_{4}=-\frac{1}{2} \lambda b_{2,2}, \quad \alpha_{5}= \pm \frac{\mathrm{i}}{2} \lambda b_{1,2}, \quad \alpha_{6}=\frac{1}{2} \lambda b_{1,1} .
\end{aligned}
$$

\section{Absolute continuity and singularity of Gaussian measures}

A probability measure $\mu$ on $\mathbb{H}$ is said to be absolutely continuous or singular if it is absolutely continuous or singular with respect to the normalized Haar measure on $\mathbb{H}$ which coincides with the Lebesgue measure on $\mathbb{R}^{3}$. The following proposition is the same as Proposition 2.1 in Pap [10]. But the proof given here is simpler, we do not use Weyl calculus.

Proposition 4.1. If $\mu$ is absolutely continuous with density $f$ then the Fourier transform $\hat{\mu}\left(\pi_{ \pm \lambda}\right)$ is an integral operator on $L^{2}(\mathbb{R})$,

$$
\left[\hat{\mu}\left(\pi_{ \pm \lambda}\right) u\right](x)=\int_{\mathbb{R}} K_{ \pm \lambda}(x, y) u(y) \mathrm{d} y
$$

with kernel function $K_{ \pm \lambda}: \mathbb{R}^{2} \rightarrow \mathbb{C}$ given by

$$
K_{ \pm \lambda}(x, y):=\frac{1}{\sqrt{\lambda}} \tilde{f}_{2,3}\left(\frac{y-x}{\sqrt{\lambda}}, \pm \sqrt{\lambda}\left(\frac{y+x}{2}\right), \pm \lambda\right),
$$

where

$$
\tilde{f}_{2,3}\left(s_{1}, \tilde{s}_{2}, \tilde{s}_{3}\right):=\int_{\mathbb{R}^{2}} \mathrm{e}^{\mathrm{i}\left(\tilde{s}_{2} s_{2}+\tilde{s}_{3} s_{3}\right)} f\left(s_{1}, s_{2}, s_{3}\right) \mathrm{d} s_{2} \mathrm{~d} s_{3}, \quad\left(s_{1}, \tilde{s}_{2}, \tilde{s}_{3}\right) \in \mathbb{R}^{3}
$$

denotes a partial Euclidean Fourier transform of $f$.

Proof. Using the definition of the Schrödinger representation we obtain

$$
\begin{aligned}
{\left[\hat{\mu}\left(\pi_{ \pm \lambda}\right) u\right](x) } & =\int_{\mathbb{R}^{3}} \mathrm{e}^{ \pm \mathrm{i}\left(\lambda s_{3}+\sqrt{\lambda} s_{2} x+\lambda s_{1} s_{2} / 2\right)} u\left(x+\sqrt{\lambda} s_{1}\right) f\left(s_{1}, s_{2}, s_{3}\right) \mathrm{d} s_{1} \mathrm{~d} s_{2} \mathrm{~d} s_{3} \\
& =\frac{1}{\sqrt{\lambda}} \int_{\mathbb{R}^{3}} \mathrm{e}^{ \pm \mathrm{i}\left(\lambda s_{3}+\sqrt{\lambda} s_{2} x+\sqrt{\lambda}(y-x) s_{2} / 2\right)} u(y) f\left(\frac{y-x}{\sqrt{\lambda}}, s_{2}, s_{3}\right) \mathrm{d} y \mathrm{~d} s_{2} \mathrm{~d} s_{3} \\
& =\int_{\mathbb{R}} K_{ \pm \lambda}(x, y) u(y) \mathrm{d} y,
\end{aligned}
$$

where

$$
\begin{aligned}
K_{ \pm \lambda}(x, y) & =\frac{1}{\sqrt{\lambda}} \int_{\mathbb{R}^{2}} \mathrm{e}^{ \pm \mathrm{i}\left(\lambda s_{3}+\sqrt{\lambda}(x+y) s_{2} / 2\right)} f\left(\frac{y-x}{\sqrt{\lambda}}, s_{2}, s_{3}\right) \mathrm{d} s_{2} \mathrm{~d} s_{3} \\
& =\frac{1}{\sqrt{\lambda}} \tilde{f}_{2,3}\left(\frac{y-x}{\sqrt{\lambda}}, \pm \sqrt{\lambda}\left(\frac{y+x}{2}\right), \pm \lambda\right) .
\end{aligned}
$$

Hence the assertion. 
The partial Euclidean Fourier transform $\tilde{f}_{2,3}$ can be obtained by the inverse Euclidean Fourier transform:

$$
\tilde{f}_{2,3}\left(s_{1}, \tilde{s}_{2}, \tilde{s}_{3}\right)=\frac{1}{2 \pi} \int_{\mathbb{R}} \mathrm{e}^{-\mathrm{i} s_{1} \tilde{s}_{1}} \tilde{f}\left(\tilde{s}_{1}, \tilde{s}_{2}, \tilde{s}_{3}\right) \mathrm{d} \tilde{s}_{1}, \quad\left(s_{1}, \tilde{s}_{2}, \tilde{s}_{3}\right) \in \mathbb{R}^{3},
$$

where $\tilde{f}$ denotes the (full) Euclidean Fourier transform of $f$ :

$$
\tilde{f}\left(\tilde{s}_{1}, \tilde{s}_{2}, \tilde{s}_{3}\right):=\int_{\mathbb{R}^{3}} \mathrm{e}^{\mathrm{i}\left(\tilde{s}_{1} s_{1}+\tilde{s}_{2} s_{2}+\tilde{s}_{3} s_{3}\right)} f\left(s_{1}, s_{2}, s_{3}\right) \mathrm{d} s_{1} \mathrm{~d} s_{2} \mathrm{~d} s_{3}
$$

for $\left(\tilde{s}_{1}, \tilde{s}_{2}, \tilde{s}_{3}\right) \in \mathbb{R}^{3}$. Moreover, $\hat{\mu}\left(\pi_{ \pm \lambda}\right)$ is a compact operator. If the density $f$ of $\mu$ belongs to the Schwarz space then $\hat{\mu}\left(\pi_{ \pm \lambda}\right)$ is a trace class (i.e., nuclear) operator.

In order to apply Proposition 4.1 we shall need the description of the set of absolutely continuous Gaussian measures on $\mathbb{H}$. Using a general result due to Siebert [14, Theorem 2] one can prove the following lemma as in Pap [10, Lemma 3.3].

Lemma 4.1. A Gaussian measure $\mu$ on $\mathbb{H}$ with parameters $(a, B)$ is either absolutely continuous or singular. More precisely, $\mu$ is absolutely continuous if $b_{1,1} b_{2,2}-b_{1,2}^{2}>0$ and singular if $b_{1,1} b_{2,2}-b_{1,2}^{2}=0$.

The next lemma describes the support of a Gaussian measure on $\mathbb{H}$.

Lemma 4.2. Let $\left(\mu_{t}\right)_{t} \geqslant 0$ be a Gaussian semigroup on $\mathbb{H}$ with infinitesimal generator $N$ given by (2.2). According to the structure of $N$ we can distinguish five different types of Gaussian semigroups:

(i) $N=Y_{0}+\frac{1}{2}\left(Y_{1}^{2}+Y_{2}^{2}+Y_{3}^{2}\right)$ with $Y_{1}, Y_{2}$ and $Y_{3}$ linearly independent. Then the semigroup is absolutely continuous and $\operatorname{supp}\left(\mu_{t}\right)=\mathbb{H}$ for all $t>0$. Moreover, $\operatorname{rank}(B)=3, b_{1,1} b_{2,2}-b_{1,2}^{2} \neq 0$.

(ii) $N=Y_{0}+\frac{1}{2}\left(Y_{1}^{2}+Y_{2}^{2}\right)$ with $Y_{1}$ and $Y_{2}$ linearly independent and $\left[Y_{1}, Y_{2}\right] \neq 0$. Then the semigroup is absolutely continuous and $\operatorname{supp}\left(\mu_{t}\right)=\mathbb{H}$ for all $t>0$. Moreover, $\operatorname{rank}(B)=2, b_{1,1} b_{2,2}-b_{1,2}^{2} \neq 0$.

(iii) $N=Y_{0}+\frac{1}{2}\left(Y_{1}^{2}+Y_{2}^{2}\right)$ with $Y_{1}$ and $Y_{2}$ linearly independent and $\left[Y_{1}, Y_{2}\right]=0$. Then the semigroup is singular, it is a Gaussian semigroup on $\mathbb{R}^{3}$ as well, and it is supported by a 'Euclidean coset' of the same closed normal subgroup, namely,

$$
\operatorname{supp}\left(\mu_{t}\right)=\exp \left(t Y_{0}+\mathbb{R} \cdot Y_{1}+\mathbb{R} \cdot Y_{2}\right)
$$

for all $t>0$. Moreover, $\operatorname{rank}(B)=2, b_{1,1} b_{2,2}-b_{1,2}^{2}=0$.

(iv) $N=Y_{0}+\frac{1}{2} Y_{1}^{2}$. Then the semigroup is singular, it is a Gaussian semigroup on $\mathbb{R}^{3}$ as well, and it is supported by a "Euclidean coset" of the same closed normal subgroup, namely,

$$
\operatorname{supp}\left(\mu_{t}\right)=\exp \left(t Y_{0}+\mathbb{R} \cdot Y_{1}+\mathbb{R} \cdot\left[Y_{0}, Y_{1}\right]\right)
$$

for all $t>0$. Moreover, $\operatorname{rank}(B)=1, b_{1,1} b_{2,2}-b_{1,2}^{2}=0$.

(v) $N=Y_{0}$. Then the semigroup is singular and consists of point measures, namely, $\mu_{t}=\varepsilon_{\exp \left(t Y_{0}\right)}$ for all $t \geqslant 0$.

Proof. From general results due to Siebert [14, Theorem 2 and Theorem 4], it follows that a Gaussian measure $\mu$ on $\mathbb{H}$ is absolutely continuous if and only if $\mathcal{G}:=\mathcal{L}\left(Y_{i},\left[Y_{j}, Y_{k}\right]: 1 \leqslant \mathrm{i} \leqslant d, 0 \leqslant j \leqslant k \leqslant d\right)=\mathbb{R}^{3}$, where $\mathcal{L}(\cdot)$ denotes the linear hull of the given vectors, and $Y_{i} \in \mathcal{H}, 0 \leqslant \mathrm{i} \leqslant d$ are described in (2.2). Moreover, the support of $\mu_{t}$ is

$$
\operatorname{supp}\left(\mu_{t}\right)=\bigcup_{n=1}^{\infty}\left(M \exp \left(\frac{t Y_{0}}{n}\right)\right)^{n} \text { for all } t>0,
$$

where $M$ is the analytic subgroup of $\mathbb{H}$ corresponding to the Lie subalgebra generated by $\left\{Y_{i}: 1 \leqslant \mathrm{i} \leqslant r\right\}$ and the bar denotes the closure in $\mathbb{H}$. Clearly $\left[Y_{i}, Y_{i}\right]=0,\left[Y_{i}, Y_{j}\right]=\left(\sigma_{1, i} \sigma_{2, j}-\sigma_{1, j} \sigma_{2, i}\right) X_{3}$ for $1 \leqslant i<j \leqslant d$ and $[Y, Z] \in \mathcal{L}\left(X_{3}\right)$ for all $Y, Z \in \mathcal{H}$.

We prove only the cases (iii) and (iv), the other cases can be proved similarly. 
In case of (iii) we have $\mathcal{G}=\mathcal{L}\left(Y_{1}, Y_{2},\left[Y_{0}, Y_{1}\right],\left[Y_{0}, Y_{2}\right]\right)$. Clearly $\left[Y_{0}, Y_{1}\right],\left[Y_{0}, Y_{2}\right] \in \mathcal{L}\left(X_{3}\right)$. Since $\left[Y_{1}, Y_{2}\right]=0$, we have $\sigma_{1,1} \sigma_{2,2}-\sigma_{1,2}^{2}=0$, so $Y_{1}$ and $Y_{2}$ are linearly dependent in their first two coordinates, thus their linear independence yields $X_{3} \in \mathcal{L}\left(Y_{1}, Y_{2}\right)$. So $\mathcal{G}=\mathcal{L}\left(Y_{1}, Y_{2}\right) \neq \mathbb{R}^{3}$, i.e., the semigroup $\left(\mu_{t}\right)_{t \geqslant 0}$ is singular.

To obtain the formula for the support of $\mu_{t}$ it is sufficient to prove that $\left(M \exp \left(\frac{t}{n} Y_{0}\right)\right)^{n}=\exp \left(t Y_{0}+\mathbb{R} \cdot Y_{1}+\mathbb{R} \cdot Y_{2}\right)$ for all $t>0$ and $n \in \mathbb{N}$, where now $M=\exp \left(\mathbb{R} \cdot Y_{1}+\mathbb{R} \cdot Y_{2}\right)$. The multiplication in $\mathbb{H}$ can be reconstructed by the help of the Campbell-Haussdorf formula

$$
\exp (X) \exp (Y)=\exp \left(X+Y+\frac{1}{2}[X, Y]\right), \quad X, Y \in \mathcal{H} .
$$

Applying induction by $n$ gives the assertion. Indeed, for $n=1$ we have $M \exp \left(t Y_{0}\right)=\exp \left(\mathbb{R} \cdot Y_{1}+\mathbb{R} \cdot Y_{2}\right) \exp \left(t Y_{0}\right)=$ $\exp \left(t Y_{0}+\mathbb{R} \cdot Y_{1}+\mathbb{R} \cdot Y_{2}\right)$, since $X_{3} \in \mathcal{L}\left(Y_{1}, Y_{2}\right)$. Suppose that $\left(M \exp \left(\frac{t}{n-1} Y_{0}\right)\right)^{n-1}=\exp \left(t Y_{0}+\mathbb{R} \cdot Y_{1}+\mathbb{R} \cdot Y_{2}\right)$ holds. Using the Campbell-Haussdorf formula and the induction hypothesis we get $\left(M \exp \left(\frac{t}{n} Y_{0}\right)\right)^{n}=\exp \left(\frac{n-1}{n} t Y_{0}+\right.$ $\left.\mathbb{R} \cdot Y_{1}+\mathbb{R} \cdot Y_{2}\right) \exp \left(\frac{t}{n} Y_{0}+\mathbb{R} \cdot Y_{1}+\mathbb{R} \cdot Y_{2}\right)$. Since $X_{3} \in \mathcal{L}\left(Y_{1}, Y_{2}\right)$ and $[Y, Z] \in \mathcal{L}\left(X_{3}\right)$ for all $Y, Z \in \mathcal{H}$, application of the Campbell-Haussdorf formula once more gives the assertion.

The case (iv) can be obtained similarly. Indeed, we have $\mathcal{G}=\mathcal{L}\left(Y_{1},\left[Y_{0}, Y_{1}\right]\right) \neq \mathbb{R}^{3}, M=\exp \left(\mathbb{R} \cdot Y_{1}\right)$, hence $\operatorname{supp}\left(\mu_{t}\right)=\exp \left(t Y_{0}+\mathbb{R} \cdot Y_{1}+\mathbb{R} \cdot\left[Y_{1}, Y_{0}\right]\right)$ for all $t>0$.

\section{Euclidean Fourier transform of a Gaussian measure and the proof of Theorem 3.1}

Now we investigate the processes $\left(W_{k}^{*}(t)\right)_{t \geqslant 0}$ and $\left(W_{k, \ell}(t)\right)_{t \geqslant 0}$ (defined in Section 2). Let $t>0$ be fixed. We prove that $W_{k}^{*}(t)$ and $W_{k, \ell}(t)$ can be constructed by the help of infinitely many independent identically distributed real random variables with standard normal distribution. Because of the self-similarity property of the Wiener process it is sufficient to prove the case $t=2 \pi$.

Lemma 5.1. Let $\left(W_{1}(s), \ldots, W_{d}(s)\right)_{s \in[0,2 \pi]}$ be a standard Wiener process in $\mathbb{R}^{d}$ on a probability space $(\Omega, \mathcal{A}, P)$. Let us consider the orthonormal basis $f_{n}(s)=(2 \pi)^{-1 / 2} \mathrm{e}^{\mathrm{i} n s}, s \in[0,2 \pi], n \in \mathbb{Z}$ in the complex Hilbert space $L^{2}([0,2 \pi])$. If $(g(s))_{s \in[0,2 \pi]}$ is an adapted, measurable, complex valued process, independent of $\left(W_{1}(s), \ldots, W_{d}(s)\right)_{s \in[0,2 \pi]}$ such that $\mathrm{E}\left(\int_{0}^{2 \pi}|g(s)|^{2} \mathrm{~d} s\right)<\infty$ then

$$
\int_{0}^{2 \pi} g(s) \mathrm{d} W_{j}(s)=\sum_{n \in \mathbb{Z}}\left\langle g, f_{n}\right\rangle \int_{0}^{2 \pi} f_{n}(s) \mathrm{d} W_{j}(s) \quad \text { a.s. }, j=1, \ldots, d,
$$

where $\langle\cdot, \cdot\rangle$ denotes the inner product in $L^{2}([0,2 \pi])$ and the convergence of the series on the right-hand side of $(5.1)$ is meant in $L^{2}(\Omega, \mathcal{A}, P)$.

Proof. Let $1 \leqslant j \leqslant d$ be arbitrary, but fixed. First we prove that the right-hand side of (5.1) is convergent in $L^{2}(\Omega, \mathcal{A}, P)$. Using that the processes $(g(s))_{s \in[0,2 \pi]}$ and $\left(W_{1}(s), \ldots, W_{d}(s)\right)_{s \in[0,2 \pi]}$ are independent of each other, for $n, m \in \mathbb{Z}, n \neq m$, we get

$$
\begin{aligned}
& \mathrm{E}\left(\left\langle g, f_{n}\right\rangle \int_{0}^{2 \pi} f_{n}(s) \mathrm{d} W_{j}(s) \overline{\left\langle g, f_{m}\right\rangle} \int_{0}^{2 \pi} \overline{f_{m}(s)} \mathrm{d} W_{j}(s)\right) \\
& =\mathrm{E}\left(\left\langle g, f_{n}\right\rangle \overline{\left\langle g, f_{m}\right\rangle}\right) \mathrm{E}\left(\int_{0}^{2 \pi} f_{n}(s) \mathrm{d} W_{j}(s) \int_{0}^{2 \pi} \overline{f_{m}(s)} \mathrm{d} W_{j}(s)\right) \\
& =\mathrm{E}\left(\left\langle g, f_{n}\right\rangle \overline{\left\langle g, f_{m}\right\rangle}\right) \int_{0}^{2 \pi} f_{n}(s) \overline{f_{m}(s)} \mathrm{d} s=0 .
\end{aligned}
$$

Using again the independence of $(g(s))_{s \in[0,2 \pi]}$ and $\left(W_{1}(s), \ldots, W_{d}(s)\right)_{s \in[0,2 \pi]}$, we have 


$$
\begin{aligned}
\mathrm{E}\left|\left\langle g, f_{n}\right\rangle \int_{0}^{2 \pi} f_{n}(s) \mathrm{d} W_{j}(s)\right|^{2} & =\mathrm{E}\left|\left\langle g, f_{n}\right\rangle\right|^{2} \mathrm{E}\left|\int_{0}^{2 \pi} f_{n}(s) \mathrm{d} W_{j}(s)\right|^{2} \\
& =\mathrm{E}\left|\left\langle g, f_{n}\right\rangle\right|^{2} \int_{0}^{2 \pi}\left|f_{n}(s)\right|^{2} \mathrm{~d} s=\mathrm{E}\left|\left\langle g, f_{n}\right\rangle\right|^{2} .
\end{aligned}
$$

Since $\mathrm{E}\left(\int_{0}^{2 \pi}|g(s)|^{2} \mathrm{~d} s\right)<\infty$, Parseval's identity in $L^{2}([0,2 \pi])$ gives us that

$$
\sum_{n \in \mathbb{Z}}\left|\left\langle g, f_{n}\right\rangle\right|^{2}=\int_{0}^{2 \pi}|g(s)|^{2} \mathrm{~d} s \quad \text { a.s. }
$$

This implies that

$$
\sum_{n \in \mathbb{Z}} \mathrm{E}\left|\left\langle g, f_{n}\right\rangle\right|^{2}=\mathrm{E} \int_{0}^{2 \pi}|g(s)|^{2} \mathrm{~d} s<\infty .
$$

Hence the right-hand side of (5.1) is convergent in $L^{2}(\Omega, \mathcal{A}, P)$.

We show now that

$$
\mathrm{E}\left|\int_{0}^{2 \pi} g(s) \mathrm{d} W_{j}(s)-\sum_{n \in \mathbb{Z}}\left\langle g, f_{n}\right\rangle \int_{0}^{2 \pi} f_{n}(s) \mathrm{d} W_{j}(s)\right|^{2}=0,
$$

which implies (5.1). We have

$$
\begin{aligned}
& \mathrm{E}\left|\int_{0}^{2 \pi} g(s) \mathrm{d} W_{j}(s)-\sum_{n \in \mathbb{Z}}\left\langle g, f_{n}\right\rangle \int_{0}^{2 \pi} f_{n}(s) \mathrm{d} W_{j}(s)\right|^{2}=\mathrm{E}\left|\int_{0}^{2 \pi} g(s) \mathrm{d} W_{j}(s)\right|^{2}+\mathrm{E}\left|\sum_{n \in \mathbb{Z}}\left\langle g, f_{n}\right\rangle \int_{0}^{2 \pi} f_{n}(s) \mathrm{d} W_{j}(s)\right|^{2} \\
& -2 \operatorname{Re} \mathrm{E}\left(\int_{0}^{2 \pi} g(s) \mathrm{d} W_{j}(s) \sum_{n \in \mathbb{Z}} \overline{\left\langle g, f_{n}\right\rangle} \int_{0}^{2 \pi} \overline{f_{n}(s)} \mathrm{d} W_{j}(s)\right)=: A_{1}+A_{2}-2 \operatorname{Re} A_{3} .
\end{aligned}
$$

Then we get

$$
\begin{aligned}
& A_{1}=\mathrm{E} \int_{0}^{2 \pi}|g(s)|^{2} \mathrm{~d} s, \\
& A_{2}=\sum_{n \in \mathbb{Z}} \mathrm{E}\left|\left\langle g, f_{n}\right\rangle \int_{0}^{2 \pi} f_{n}(s) \mathrm{d} W_{j}(s)\right|^{2}=\sum_{n \in \mathbb{Z}} \mathrm{E}\left|\left\langle g, f_{n}\right\rangle\right|^{2}=\mathrm{E} \int_{0}^{2 \pi}|g(s)|^{2} \mathrm{~d} s, \\
& A_{3}=\sum_{n \in \mathbb{Z}} \mathrm{E}\left(\int_{0}^{2 \pi} g(s) \mathrm{d} W_{j}(s) \overline{\left\langle g, f_{n}\right\rangle} \int_{0}^{2 \pi} \overline{f_{n}(s)} \mathrm{d} W_{j}(s)\right) .
\end{aligned}
$$

Let us denote the $\sigma$-algebra generated by the process $(g(s))_{s \in[0,2 \pi]}$ by $\mathcal{F}(g)$. Then we obtain

$$
\begin{aligned}
A_{3} & =\sum_{n \in \mathbb{Z}} \mathrm{EE}\left(\int_{0}^{2 \pi} g(s) \mathrm{d} W_{j}(s) \overline{\left\langle g, f_{n}\right\rangle} \int_{0}^{2 \pi} \overline{f_{n}(s)} \mathrm{d} W_{j}(s) \mid \mathcal{F}(g)\right) \\
& =\sum_{n \in \mathbb{Z}} \mathrm{E}\left(\overline{\left\langle g, f_{n}\right\rangle} \mathrm{E}\left(\int_{0}^{2 \pi} g(s) \mathrm{d} W_{j}(s) \int_{0}^{2 \pi} \overline{f_{n}(s)} \mathrm{d} W_{j}(s) \mid \mathcal{F}(g)\right)\right)
\end{aligned}
$$




$$
=\sum_{n \in \mathbb{Z}} \mathrm{E}\left(\overline{\left\langle g, f_{n}\right\rangle} \int_{0}^{2 \pi} g(s) \overline{f_{n}(s)} \mathrm{d} s\right)=\sum_{n \in \mathbb{Z}} \mathrm{E}\left|\left\langle g, f_{n}\right\rangle\right|^{2}=\mathrm{E} \int_{0}^{2 \pi}|g(s)|^{2} \mathrm{~d} s .
$$

Hence the assertion.

The next statement is a generalization of Section 1.2 in Chaleyat-Maurel [3].

Lemma 5.2. Let $\left(W_{1}(s), \ldots, W_{d}(s)\right)_{s \in[0,2 \pi]}$ be a standard Wiener process in $\mathbb{R}^{d}$. Then there exist random variables $a_{n}^{(j)}, b_{n}^{(j)}, n \in \mathbb{N}, j=1, \ldots, d$, with standard normal distribution, independent of each other and of the random variable $\left(W_{1}(2 \pi), \ldots, W_{d}(2 \pi)\right)$ such that the following constructions hold

$$
\begin{aligned}
& W_{j, k}(2 \pi)=\sum_{n=1}^{\infty} \frac{1}{n}\left[b_{n}^{(j)}\left(a_{n}^{(k)}-\frac{1}{\sqrt{\pi}} W_{k}(2 \pi)\right)-b_{n}^{(k)}\left(a_{n}^{(j)}-\frac{1}{\sqrt{\pi}} W_{j}(2 \pi)\right)\right] \text { a.s. }, \\
& W_{\ell}^{*}(2 \pi)=-2 \sqrt{\pi} \sum_{n=1}^{\infty} \frac{b_{n}^{(\ell)}}{n} \text { a.s. }
\end{aligned}
$$

for all $1 \leqslant j<k \leqslant d$ and $\ell=1, \ldots, d$, where the series on the right-hand sides of (5.2) and (5.3) are convergent almost surely.

Proof. Retain the notations of Lemma 5.1 and let us denote $c_{n}^{(j)}:=\int_{0}^{2 \pi} \overline{f_{n}(s)} \mathrm{d} W_{j}(s), n \in \mathbb{Z}, j=1, \ldots, d$. Then $c_{n}^{(j)}$, $n \in \mathbb{Z}, j=1, \ldots, d$, are independent identically distributed complex random variables with standard normal distribution, i.e., the decompositions $c_{n}^{(j)}=\frac{a_{n}^{(j)}+\mathrm{i} b_{n}^{(j)}}{\sqrt{2}}, n \in \mathbb{Z}, j=1, \ldots, d$, hold with independent identically distributed real random variables $a_{n}^{(j)}, b_{n}^{(j)}, n \in \mathbb{Z}, j=1, \ldots, d$, having standard normal distribution. Specifying $g$ as the indicator function $\mathbb{1}_{[0, t]}$ of the interval $[0, t](t \in[0,2 \pi])$ in Lemma 5.1, we have for all $t \in[0,2 \pi]$

$$
W_{\ell}(t)=\sum_{n \in \mathbb{Z}, n \neq 0} c_{-n}^{(\ell)} \frac{\mathrm{i}}{n}\left(f_{-n}(t)-f_{0}(t)\right)+\frac{c_{0}^{(\ell)} t}{\sqrt{2 \pi}} \quad \text { a.s., } \ell=1, \ldots, d .
$$

In fact, there is a set $\Omega_{0}$ with $P\left(\Omega_{0}\right)=0$ such that (5.4) holds for all $\omega \notin \Omega_{0}$ and for almost every $t \in[0,2 \pi]$ (see, e.g., Ash [1, p. 107, Problem 4]). Applying (5.1) with $g=W_{k}$ and the construction (5.4), Chaleyat-Maurel [3] showed that (5.2) holds. Choosing $g(s)=s \mathbb{1}_{[0, t]}(s)(t \in[0,2 \pi])$ in Lemma 5.1 it can be easily checked that

$$
\int_{0}^{t} s \mathrm{~d} W_{\ell}(s)=\sum_{n \in \mathbb{Z}, n \neq 0} \frac{c_{-n}^{(\ell)}(\mathrm{i} n t+1)}{n^{2}} f_{-n}(t)-\sum_{n \in \mathbb{Z}, n \neq 0} \frac{c_{-n}^{(\ell)}}{n^{2}} f_{0}(t)+c_{0}^{(\ell)} \frac{t^{2}}{2 \sqrt{2 \pi}} \text { a.s. }
$$

By Itô's formula we get $W_{\ell}^{*}(t)=\frac{1}{2} t W_{\ell}(t)-\int_{0}^{t} s \mathrm{~d} W_{\ell}(s)$. Using the construction (5.4) of $W_{\ell}(t)$ and the definition of $c_{n}^{(\ell)}$ a simple computation shows that (5.3) holds. By Lemma 5.1 the series in the constructions (5.2), (5.3) and (5.4) are convergent in $L^{2}(\Omega, \mathcal{A}, P)$. Since the summands in each series are independent of each other, Lévy's theorem implies that they are convergent almost surely, too.

Taking into account Proposition 4.1 and the representation of a Gaussian semigroup $\left(\mu_{t}\right)_{t \geqslant 0}$ by the process $(Z(t))_{t \geqslant 0}$ (given in Section 2), in order to prove Theorem 3.1 we need the joint (Euclidean) Fourier transform of the 9-dimensional random vector

$$
\left(W_{1}(t), W_{2}(t), W_{3}(t), W_{1}^{*}(t), W_{2}^{*}(t), W_{3}^{*}(t), W_{1,2}(t), W_{1,3}(t), W_{2,3}(t)\right) .
$$

Proposition 5.1. The Fourier transform $\widetilde{F}_{t}: \mathbb{R}^{9} \rightarrow \mathbb{C}$ of the random vector (5.5) is

$$
\begin{aligned}
& \widetilde{F}_{t}\left(\eta_{1}, \eta_{2}, \eta_{3}, \zeta_{1}, \zeta_{2}, \zeta_{3}, \xi_{1,2}, \xi_{1,3}, \xi_{2,3}\right) \\
& \quad=\frac{1}{\cosh (t\|\tilde{\xi}\| / 2)} \exp \left\{-\frac{t^{3}}{4\|\tilde{\xi}\|^{2}}\left(\frac{1}{6}-\frac{2 \kappa}{t^{2}\|\tilde{\xi}\|^{2}}\right)\langle\tilde{\xi}, \zeta\rangle^{2}+\frac{\|\tilde{\xi}\|^{2}\|\tilde{\eta}\|^{2}+\kappa\langle\tilde{\xi}, \tilde{\eta}\rangle^{2}-t \kappa(1+\kappa)\|\zeta\|^{2}}{2(1+\kappa)\|\tilde{\xi}\|^{2}}\right\}
\end{aligned}
$$


for $\tilde{\xi}:=\left(\xi_{2,3},-\xi_{1,3}, \xi_{1,2}\right)^{\top} \in \mathbb{R}^{3}$ with $\tilde{\xi} \neq 0$, where $\eta:=\left(\eta_{1}, \eta_{2}, \eta_{3}\right)^{\top} \in \mathbb{R}^{3}, \zeta:=\left(\zeta_{1}, \zeta_{2}, \zeta_{3}\right)^{\top} \in \mathbb{R}^{3}$, and

$$
\kappa:=\frac{t\|\tilde{\xi}\|}{2} \operatorname{coth}\left(\frac{t\|\tilde{\xi}\|}{2}\right)-1, \quad \tilde{\eta}:=\frac{\sqrt{t} \kappa}{\|\tilde{\xi}\|^{2}} \xi \zeta+\mathrm{i} \sqrt{t} \eta, \quad \xi:=\left[\begin{array}{ccc}
0 & \xi_{1,2} & \xi_{1,3} \\
-\xi_{1,2} & 0 & \xi_{2,3} \\
-\xi_{1,3} & -\xi_{2,3} & 0
\end{array}\right] .
$$

(Here $\|\cdot\|$ and $\langle\cdot, \cdot\rangle$ denote the Euclidean norm and scalar product, respectively.)

To calculate the Fourier transform of (5.5) we will use the constructions of the processes $\left(W_{k}^{*}(t)\right)_{t \geqslant 0}$ and $\left(W_{k, \ell}(t)\right)_{\geqslant 0}$ (see Lemma 5.2) and the following lemma.

Lemma 5.3. Let $X$ be a $k$-dimensional real random vector with standard normal distribution. Then we have

$$
\operatorname{Eexp}\{\langle\tilde{\eta}, X\rangle-s\langle B X, X\rangle\}=\frac{1}{\sqrt{\operatorname{det}(I+2 s B)}} \exp \left\{\frac{1}{2}\left\langle\tilde{\eta},(I+2 s B)^{-1} \tilde{\eta}\right\rangle\right\},
$$

for all $\tilde{\eta} \in \mathbb{C}^{k}, s \in \mathbb{R}^{+}$and real symmetric positive semidefinite matrices $B$. (Here I denotes the $k \times k$ identity matrix.)

Proof. Consider the decomposition $B=U \Lambda U^{\top}$, where $\Lambda$ is the $k \times k$ diagonal matrix containing the eigenvalues of $B$ in its diagonal and $U$ is an orthogonal matrix. Then the random vector $Y:=U^{\top} X$ has also a standard normal distribution. This implies that

$$
\begin{aligned}
\mathrm{E} \exp \{\langle\tilde{\eta}, X\rangle-s\langle B X, X\rangle\} & =\operatorname{E} \exp \{\langle\tilde{\eta}, U Y\rangle-s\langle\Lambda Y, Y\rangle\} \\
& =\frac{1}{\sqrt{(2 \pi)^{k}}} \int_{\mathbb{R}^{k}} \exp \left\{\langle\tilde{\eta}, U y\rangle-s\langle\Lambda y, y\rangle-\frac{1}{2}\langle y, y\rangle\right\} \mathrm{d} y,
\end{aligned}
$$

where $y=\left(y_{1}, \ldots, y_{k}\right)^{\top} \in \mathbb{R}^{k}$. Let $\lambda_{1}, \ldots, \lambda_{k}$ denote the eigenvalues of the matrix $B$. A simple computation shows that

$$
\begin{aligned}
\langle\tilde{\eta}, U y\rangle-s\langle\Lambda y, y\rangle-\frac{1}{2}\langle y, y\rangle & =-\sum_{j=1}^{k}\left(s \lambda_{j}+\frac{1}{2}\right) y_{j}^{2}+\sum_{j=1}^{k}\left(U^{\top} \operatorname{Re} \tilde{\eta}\right)_{j} y_{j}+\mathrm{i} \sum_{j=1}^{k}\left(U^{\top} \operatorname{Im} \tilde{\eta}\right)_{j} y_{j} \\
& =\mathrm{i} \sum_{j=1}^{k}\left(U^{\top} \operatorname{Im} \tilde{\eta}\right)_{j} y_{j}-\sum_{j=1}^{k} \frac{1+2 s \lambda_{j}}{2}\left(y_{j}-\frac{\left(U^{\top} \operatorname{Re} \tilde{\eta}\right)_{j}}{1+2 s \lambda_{j}}\right)^{2}+\sum_{j=1}^{k} \frac{\left(U^{\top} \operatorname{Re} \tilde{\eta}\right)_{j}^{2}}{2\left(1+2 s \lambda_{j}\right)} .
\end{aligned}
$$

Using the well-known formula for the Fourier transform of a standard normal distribution

$$
\int_{\mathbb{R}} \exp \left\{\mathrm{i} x t-\frac{(x-m)^{2}}{2 \sigma^{2}}\right\} \mathrm{d} x=\sqrt{2 \pi} \sigma \exp \left\{\mathrm{i} m t-\frac{1}{2} \sigma^{2} t^{2}\right\},
$$

for all $t, m \in \mathbb{R}$ and $\sigma>0$, we obtain

$$
\begin{aligned}
& E \exp \{\langle\tilde{\eta}, X\rangle-s\langle B X, X\rangle\} \\
& =\frac{1}{\sqrt{\prod_{j=1}^{k}\left(1+2 s \lambda_{j}\right)}} \exp \left\{\mathrm{i} \sum_{j=1}^{k} \frac{\left(U^{\top} \operatorname{Re} \tilde{\eta}\right)_{j}\left(U^{\top} \operatorname{Im} \tilde{\eta}\right)_{j}}{1+2 s \lambda_{j}}-\sum_{j=1}^{k} \frac{\left(U^{\top} \operatorname{Im} \tilde{\eta}\right)_{j}^{2}}{2\left(1+2 s \lambda_{j}\right)}+\sum_{j=1}^{k} \frac{\left(U^{\top} \operatorname{Re} \tilde{\eta}\right)_{j}^{2}}{2\left(1+2 s \lambda_{j}\right)}\right\} .
\end{aligned}
$$

Hence the assertion.

Proof of Proposition 5.1. Because of the self-similarity property of the Wiener process, the random vectors $\left(W_{k}(t), W_{\ell}^{*}(t), W_{p, q}(t): 1 \leqslant k, \ell \leqslant d, 1 \leqslant p<q \leqslant d\right)$ and $\left(c^{-1 / 2} W_{k}(c t), c^{-3 / 2} W_{\ell}^{*}(c t), c^{-1} W_{p, q}(c t): 1 \leqslant k, \ell \leqslant d\right.$, $1 \leqslant p<q \leqslant d)$ have the same distribution for all $t \geqslant 0$ and $c>0$. Hence

$$
\begin{aligned}
& \widetilde{F}_{t}\left(\eta_{1}, \eta_{2}, \eta_{3}, \zeta_{1}, \zeta_{2}, \zeta_{3}, \xi_{1,2}, \xi_{1,3}, \xi_{2,3}\right) \\
& \quad=\widetilde{F}_{2 \pi}\left(\sqrt{\frac{t}{2 \pi}} \eta_{1}, \sqrt{\frac{t}{2 \pi}} \eta_{2}, \sqrt{\frac{t}{2 \pi}} \eta_{3},\left(\frac{t}{2 \pi}\right)^{3 / 2} \zeta_{1},\left(\frac{t}{2 \pi}\right)^{3 / 2} \zeta_{2},\left(\frac{t}{2 \pi}\right)^{3 / 2} \zeta_{3}, \frac{t}{2 \pi} \xi_{1,2}, \frac{t}{2 \pi} \xi_{1,3}, \frac{t}{2 \pi} \xi_{2,3}\right),
\end{aligned}
$$


so it is sufficient to determine $\widetilde{F}_{2 \pi}$. By the definition of the Fourier transform we get

$$
\begin{aligned}
& \widetilde{F}_{2 \pi}\left(\eta_{1}, \eta_{2}, \eta_{3}, \zeta_{1}, \zeta_{2}, \zeta_{3}, \xi_{1,2}, \xi_{1,3}, \xi_{2,3}\right) \\
& \quad=\operatorname{E} \exp \left\{\mathrm{i}\left(\sum_{j=1}^{3} \eta_{j} W_{j}(2 \pi)+\sum_{j=1}^{3} \zeta_{j} W_{j}^{*}(2 \pi)+\sum_{1 \leqslant j<k \leqslant 3} \xi_{j, k} W_{j, k}(2 \pi)\right)\right\} .
\end{aligned}
$$

For abbreviation let $\widetilde{F}_{2 \pi}$ denote $\widetilde{F}_{2 \pi}\left(\eta_{1}, \eta_{2}, \eta_{3}, \zeta_{1}, \zeta_{2}, \zeta_{3}, \xi_{1,2}, \xi_{1,3}, \xi_{2,3}\right)$. Define the random vector $\chi:=\left(\chi_{1}, \chi_{2}, \chi_{3}\right)^{\top}$ by

$$
\begin{aligned}
& \chi_{1}:=-\xi_{1,2} \frac{1}{\sqrt{\pi}} W_{2}(2 \pi)-\xi_{1,3} \frac{1}{\sqrt{\pi}} W_{3}(2 \pi)-2 \sqrt{\pi} \zeta_{1}, \\
& \chi_{2}:=\xi_{1,2} \frac{1}{\sqrt{\pi}} W_{1}(2 \pi)-\xi_{2,3} \frac{1}{\sqrt{\pi}} W_{3}(2 \pi)-2 \sqrt{\pi} \zeta_{2}, \\
& \chi_{3}:=\xi_{1,3} \frac{1}{\sqrt{\pi}} W_{1}(2 \pi)+\xi_{2,3} \frac{1}{\sqrt{\pi}} W_{2}(2 \pi)-2 \sqrt{\pi} \zeta_{3} .
\end{aligned}
$$

Substituting the expressions (5.2), (5.3) for $W_{j, k}(2 \pi)$ and $W_{\ell}^{*}(2 \pi)$ into the formula (5.9), taking conditional expectation with respect to $\left\{W_{j}(2 \pi), a_{n}^{(j)}, 1 \leqslant j \leqslant 3, n \geqslant 1\right\}$, and using the identity $\mathrm{E}(\mathrm{E}(X \mid Y))=\mathrm{E} X$ (where $X, Y$ random variables, $\mathrm{E}|X|<\infty)$, we obtain

$$
\begin{aligned}
\widetilde{F}_{2 \pi}= & \mathrm{E}\left[\exp \left\{\mathrm{i}\left(\eta_{1} W_{1}(2 \pi)+\eta_{2} W_{2}(2 \pi)+\eta_{3} W_{3}(2 \pi)\right)\right\}\right. \\
& \left.\times \mathrm{E}\left(\exp \left\{\mathrm{i} \sum_{n=1}^{\infty} \frac{1}{n}\left\langle\xi \cdot a_{n}+\chi, b_{n}\right\rangle\right\} \mid W_{j}(2 \pi), a_{n}^{(j)}, 1 \leqslant j \leqslant 3, n \geqslant 1\right)\right],
\end{aligned}
$$

where $a_{n}:=\left(a_{n}^{(1)}, a_{n}^{(2)}, a_{n}^{(3)}\right)^{\top}$ and $b_{n}:=\left(b_{n}^{(1)}, b_{n}^{(2)}, b_{n}^{(3)}\right)^{\top}$. Taking into account that $b_{n}^{(1)}, b_{n}^{(2)}, b_{n}^{(3)}$ are independent of the condition above and of each other for all $n \in \mathbb{N}$, using the dominated convergence theorem and the explicit formula for the Fourier transform of a standard normal distribution we get

$$
\widetilde{F}_{2 \pi}=\mathrm{E}\left[\exp \left\{\mathrm{i}\left(\eta_{1} W_{1}(2 \pi)+\eta_{2} W_{2}(2 \pi)+\eta_{3} W_{3}(2 \pi)\right)\right\} \prod_{n=1}^{\infty} \exp \left\{-\frac{1}{2 n^{2}}\left\|\xi \cdot a_{n}+\chi\right\|^{2}\right\}\right] .
$$

Since $\xi$ is a skew symmetric matrix, there exists an orthogonal matrix $M=\left(m_{j, k}\right)_{1 \leqslant j, k \leqslant 3}$ such that

$$
M^{\top} \xi M=\left[\begin{array}{ccc}
0 & p & 0 \\
-p & 0 & 0 \\
0 & 0 & 0
\end{array}\right]=: P
$$

The orthogonality of $M$ implies $M^{-1}=M^{\top}$, hence $\xi M=M P$. We have

$$
M P=\left[\begin{array}{ccc}
-p m_{1,2} & p m_{1,1} & 0 \\
-p m_{2,2} & p m_{2,1} & 0 \\
-p m_{3,2} & p m_{3,1} & 0
\end{array}\right]=\left[-p \mathbf{m}_{2}, p \mathbf{m}_{1}, 0\right],
$$

where $\mathbf{m}_{i}, i=1,2,3$, denotes the column vectors of $M$, that is, $M=\left[\mathbf{m}_{1}, \mathbf{m}_{2}, \mathbf{m}_{3}\right]$. Obviously, $\xi M=\left[\xi \mathbf{m}_{1}, \xi \mathbf{m}_{2}\right.$, $\left.\xi \mathbf{m}_{3}\right]$, hence $\xi \mathbf{m}_{1}=-p \mathbf{m}_{2}, \xi \mathbf{m}_{2}=p \mathbf{m}_{1}, \xi \mathbf{m}_{3}=0$. Taking into account that $M$ is orthogonal, we have $\left\|\mathbf{m}_{3}\right\|=1$, hence

$$
\mathbf{m}_{3}= \pm \frac{1}{\sqrt{\xi_{1,2}^{2}+\xi_{1,3}^{2}+\xi_{2,3}^{2}}}\left(\xi_{2,3},-\xi_{1,3}, \xi_{1,2}\right)^{\top} .
$$

Moreover, $\xi^{2} \mathbf{m}_{1}=\xi\left(\xi \mathbf{m}_{1}\right)=\xi\left(-p \mathbf{m}_{2}\right)=-p^{2} \mathbf{m}_{1}$. The only nonzero eigenvalue of $\xi^{2}$ is $-\left(\xi_{1,2}^{2}+\xi_{1,3}^{2}+\xi_{2,3}^{2}\right)$, hence $p= \pm \sqrt{\xi_{1,2}^{2}+\xi_{1,3}^{2}+\xi_{2,3}^{2}}$, and $M$ can be chosen such that $\mathbf{m}_{3}=\tilde{\xi} /\|\tilde{\xi}\|, p=\|\tilde{\xi}\|$, and thus

$$
\left\langle\mathbf{m}_{1}, u\right\rangle^{2}+\left\langle\mathbf{m}_{2}, u\right\rangle^{2}=\left\|M^{\top} u\right\|^{2}-\left\langle\mathbf{m}_{3}, u\right\rangle^{2}=\|u\|^{2}-\frac{1}{\|\tilde{\xi}\|^{2}}\langle\tilde{\xi}, u\rangle^{2},
$$


for all $u \in \mathbb{R}^{3}$. We also get

$$
-\xi^{2}=M\left[\begin{array}{ccc}
\|\tilde{\xi}\|^{2} & 0 & 0 \\
0 & \|\tilde{\xi}\|^{2} & 0 \\
0 & 0 & 0
\end{array}\right] M^{\top}=: M \Lambda M^{\top}
$$

To continue the calculation of the Fourier transform of (5.5) we take conditional expectation with respect to $\left\{W_{1}(2 \pi), W_{2}(2 \pi), W_{3}(2 \pi)\right\}$. A special case of Lemma 5.3 is that

$$
\left.\mathrm{E} \exp \left\{-s \sum_{j=1}^{n} Y_{j}^{2}\right\}=\frac{1}{\sqrt{\operatorname{det}(I+2 s D)}} \exp \left\{\left(2 s^{2} D^{1 / 2}(I+2 s D)^{-1} D^{1 / 2}-s I\right) m, m\right\rangle\right\}
$$

for all $s \in \mathbb{R}^{+}$, where $Y=\left(Y_{1}, \ldots, Y_{k}\right)^{\top}$ is a $k$-dimensional random variable with normal distribution such that $\mathrm{E} Y=m$ and $\operatorname{Var} Y=D$. Applying this formula for $Y=\xi \cdot a_{n}+\chi$ with $s=\left(2 n^{2}\right)^{-1}, m=\chi$ and $D=\xi \cdot \xi^{\top}=$ $-\xi^{2}=M \Lambda M^{\top}$ we get

$$
\begin{aligned}
\widetilde{F}_{2 \pi}= & \mathrm{E}\left[\exp \left\{\mathrm{i}\left(\eta_{1} W_{1}(2 \pi)+\eta_{2} W_{2}(2 \pi)+\eta_{3} W_{3}(2 \pi)\right)\right\}\right. \\
& \left.\times \prod_{n=1}^{\infty} \frac{1}{\sqrt{\operatorname{det}\left(I+n^{-2} \Lambda\right)}} \exp \left\{\frac{1}{2}\left\langle\left(n^{-4} \sqrt{\Lambda}\left(I+n^{-2} \Lambda\right)^{-1} \sqrt{\Lambda}-n^{-2} I\right) M^{-1} \chi, M^{-1} \chi\right\rangle\right\}\right] .
\end{aligned}
$$

Clearly $\operatorname{det}\left(I+n^{-2} \Lambda\right)=\left(1+n^{-2}\|\tilde{\xi}\|^{2}\right)^{2}$. Using that

$$
\prod_{k=1}^{\infty} \frac{k^{2} \pi^{2}}{k^{2} \pi^{2}+x^{2}}=\frac{x}{\sinh x}, \quad x \operatorname{coth} x-1=x^{2} \sum_{k=1}^{\infty} \frac{2}{k^{2} \pi^{2}+x^{2}}, \quad x \in \mathbb{R}
$$

(see [5], formulas 1.431 and 1.421), the identity (5.10) and the fact that $\langle\tilde{\xi}, \chi\rangle^{2}=4 \pi\langle\zeta, \tilde{\xi}\rangle^{2}$ we obtain

$$
\begin{aligned}
\widetilde{F}_{2 \pi}= & \frac{\pi\|\tilde{\xi}\|}{\sinh (\pi\|\tilde{\xi}\|)} \exp \left\{-\frac{\pi^{3}}{\|\tilde{\xi}\|^{2}}\left(\frac{1}{3}-\frac{\kappa}{\pi^{2}\|\tilde{\xi}\|^{2}}\right)\langle\zeta, \tilde{\xi}\rangle^{2}\right\} \\
& \times \operatorname{Eexp}\left\{i\left(\eta_{1} W_{1}(2 \pi)+\eta_{2} W_{2}(2 \pi)+\eta_{3} W_{3}(2 \pi)\right)-\frac{\kappa}{4\|\tilde{\xi}\|^{2}}\|\chi\|^{2}\right\},
\end{aligned}
$$

where $\kappa=\pi\|\tilde{\xi}\| \operatorname{coth}(\pi\|\tilde{\xi}\|)-1$. A simple computation shows that

$$
\begin{aligned}
\|\chi\|^{2}= & \frac{1}{\pi}\left(\left(\xi_{1,2}^{2}+\xi_{1,3}^{2}\right) W_{1}^{2}(2 \pi)+\left(\xi_{1,2}^{2}+\xi_{2,3}^{2}\right) W_{2}^{2}(2 \pi)+\left(\xi_{2,3}^{2}+\xi_{1,3}^{2}\right) W_{3}^{2}(2 \pi)\right)+4 \pi\|\zeta\|^{2} \\
& +\frac{2}{\pi}\left(\xi_{1,3} \xi_{2,3} W_{1}(2 \pi) W_{2}(2 \pi)-\xi_{1,2} \xi_{2,3} W_{1}(2 \pi) W_{3}(2 \pi)+\xi_{1,2} \xi_{1,3} W_{2}(2 \pi) W_{3}(2 \pi)\right) \\
& -4\left(\xi_{1,2} \zeta_{2}+\xi_{1,3} \zeta_{3}\right) W_{1}(2 \pi)+4\left(\xi_{1,2} \zeta_{1}-\xi_{2,3} \zeta_{3}\right) W_{2}(2 \pi)+4\left(\xi_{1,3} \zeta_{1}+\xi_{2,3} \zeta_{2}\right) W_{3}(2 \pi) .
\end{aligned}
$$

Using Lemma 5.3 with

$$
\tilde{\eta}=\frac{\sqrt{2 \pi} \kappa}{\|\tilde{\xi}\|^{2}} \xi \zeta+\mathrm{i} \sqrt{2 \pi} \eta, \quad B:=-2 \xi^{2}, \quad s=\frac{\kappa}{4\|\tilde{\xi}\|^{2}}
$$

and taking into account that $\sqrt{\operatorname{det}(I+2 s B)}=1+\kappa$ we conclude

$$
\begin{aligned}
\widetilde{F}_{2 \pi}= & \frac{\pi\|\tilde{\xi}\|}{(1+\kappa) \sinh (\pi\|\tilde{\xi}\|)} \exp \left\{-\frac{\pi^{3}}{\|\tilde{\xi}\|^{2}}\left(\frac{1}{3}-\frac{\kappa}{\pi^{2}\|\tilde{\xi}\|^{2}}\right)\langle\zeta, \tilde{\xi}\rangle^{2}\right\} \\
& \times \exp \left\{-\frac{\pi \kappa}{\|\tilde{\xi}\|^{2}}\|\zeta\|^{2}+\frac{1}{2}\left\langle\tilde{\eta},\left(I-\frac{\kappa}{\|\tilde{\xi}\|^{2}} \xi^{2}\right)^{-1} \tilde{\eta}\right)\right\}
\end{aligned}
$$

Using (5.10) we get 


$$
\left\langle\tilde{\eta},\left(I-\frac{\kappa}{\|\tilde{\xi}\|^{2}} \xi^{2}\right)^{-1} \tilde{\eta}\right\rangle=\frac{1}{1+\kappa}\|\tilde{\eta}\|^{2}+\frac{\kappa}{1+\kappa} \frac{\langle\tilde{\xi}, \tilde{\eta}\rangle^{2}}{\|\tilde{\xi}\|^{2}} .
$$

Hence the assertion.

Proof of Theorem 3.1. We prove only the case $\operatorname{rank}(B)=3$. The cases $\operatorname{rank}(B)=1$ and $\operatorname{rank}(B)=2$ can be handled in a similar way. In case $\operatorname{rank}(B)=3$ the measure $\mu$ is absolutely continuous and so Proposition 4.1 implies that the partial Euclidean Fourier transform $\tilde{f}_{2,3}$ of the measure $\mu$ has to be calculated in order to obtain the Fourier transform $\hat{\mu}\left(\pi_{ \pm \lambda}\right)$. Let $\left(\mu_{t}\right)_{t \geqslant 0}$ be a Gaussian semigroup such that $\mu_{1}=\mu$ and let $\rho_{1}:=\sigma_{1,1} \sigma_{2,2}-\sigma_{1,2} \sigma_{2,1}, \rho_{2}:=$ $\sigma_{1,1} \sigma_{2,3}-\sigma_{1,3} \sigma_{2,1}, \rho_{3}:=\sigma_{1,2} \sigma_{2,3}-\sigma_{1,3} \sigma_{2,2}$ by definition. In case $\operatorname{rank}(B)=3$, the representation of $\left(\mu_{t}\right)_{t \geqslant 0}$ by the process $(Z(t))_{t \geqslant 0}$ (see Section 2$)$ gives us

$$
\begin{aligned}
& Z_{1}(1)=a_{1}+\sum_{k=1}^{3} \sigma_{1, k} W_{k}(1), \quad Z_{2}(1)=a_{2}+\sum_{k=1}^{3} \sigma_{2, k} W_{k}(1), \\
& Z_{3}(1)=a_{3}+\sum_{k=1}^{3} \sigma_{3, k} W_{k}(1)+\sum_{k=1}^{3}\left(a_{2} \sigma_{1, k}-a_{1} \sigma_{2, k}\right) W_{k}^{*}(1)+\rho_{1} W_{1,2}(1)+\rho_{2} W_{1,3}(1)+\rho_{3} W_{2,3}(1) .
\end{aligned}
$$

This implies that the (full) Euclidean Fourier transform of the measure $\mu$ is

$$
\begin{aligned}
& \tilde{f}\left(\tilde{s}_{1}, \tilde{s}_{2}, \tilde{s}_{3}\right)= \operatorname{Exp}\left\{\operatorname{i}\left(\tilde{s}_{1} Z_{1}(1)+\tilde{s}_{2} Z_{2}(1)+\tilde{s}_{3} Z_{3}(1)\right)\right\}=\exp \left\{\mathrm{i}\left(\tilde{s}_{1} a_{1}+\tilde{s}_{2} a_{2}+\tilde{s}_{3} a_{3}\right)\right\} \\
& \times \operatorname{E} \exp \left\{\mathrm { i } \left(\sum_{k=1}^{3}\left(\sigma_{1, k} \tilde{s}_{1}+\sigma_{2, k} \tilde{s}_{2}+\sigma_{3, k} \tilde{s}_{3}\right) W_{k}(1)+\sum_{k=1}^{3}\left(a_{2} \sigma_{1, k}-a_{1} \sigma_{2, k}\right) \tilde{s}_{3} W_{k}^{*}(1)\right.\right. \\
&\left.\left.\quad+\tilde{s}_{3} \rho_{1} W_{1,2}(1)+\tilde{s}_{3} \rho_{2} W_{1,3}(1)+\tilde{s}_{3} \rho_{3} W_{2,3}(1)\right)\right\}
\end{aligned}
$$

Proposition 4.1 shows that we may suppose $\tilde{s}_{3} \neq 0$. Using Proposition 5.1 and the facts that

$$
\begin{aligned}
& \sum_{k=1}^{d}\left(a_{2} \sigma_{1, k}-a_{1} \sigma_{2, k}\right)^{2}=b_{2,2} a_{1}^{2}-2 b_{1,2} a_{1} a_{2}+b_{1,1} a_{2}^{2}, \quad d=1,2,3, \\
& \rho_{1}\left(a_{1} \sigma_{2,3}-a_{2} \sigma_{1,3}\right)-\rho_{2}\left(a_{1} \sigma_{2,2}-a_{2} \sigma_{1,2}\right)+\rho_{3}\left(a_{1} \sigma_{2,1}-a_{2} \sigma_{1,1}\right)=0, \\
& \delta^{2}=\rho_{1}^{2}+\rho_{2}^{2}+\rho_{3}^{2},
\end{aligned}
$$

we get

$$
\begin{gathered}
\tilde{f}\left(\tilde{s}_{1}, \tilde{s}_{2}, \tilde{s}_{3}\right)=\frac{1}{\cosh \left(\left|\tilde{s}_{3}\right| \delta / 2\right)} \exp \left\{\mathrm{i}\left(\tilde{s}_{1} a_{1}+\tilde{s}_{2} a_{2}+\tilde{s}_{3} a_{3}\right)-\frac{\kappa}{2 \delta^{2}}\left(b_{2,2} a_{1}^{2}-2 b_{1,2} a_{1} a_{2}+b_{1,1} a_{2}^{2}\right)\right. \\
\left.+\frac{1}{2(1+\kappa)}\|\tilde{\eta}\|^{2}+\frac{\kappa}{2(1+\kappa)} \frac{\langle\tilde{\xi}, \tilde{\eta}\rangle^{2}}{\delta^{2}}\right\},
\end{gathered}
$$

where

$$
\kappa=\frac{\left|\tilde{s}_{3}\right| \delta}{2} \operatorname{coth}\left(\frac{\left|\tilde{s}_{3}\right| \delta}{2}\right)-1, \quad \tilde{\eta}=-\frac{\kappa}{\delta^{2}}\left(v_{1}, v_{2}, v_{3}\right)^{\top}+\mathrm{i} \Sigma^{\top} \tilde{s}
$$

with

$$
\begin{aligned}
& v_{1}=\rho_{1}\left(a_{1} \sigma_{2,2}-a_{2} \sigma_{1,2}\right)+\rho_{2}\left(a_{1} \sigma_{2,3}-a_{2} \sigma_{1,3}\right), \\
& v_{2}=-\rho_{1}\left(a_{1} \sigma_{2,1}-a_{2} \sigma_{1,1}\right)+\rho_{3}\left(a_{1} \sigma_{2,3}-a_{2} \sigma_{1,3}\right), \\
& v_{3}=-\rho_{2}\left(a_{1} \sigma_{2,1}-a_{2} \sigma_{1,1}\right)-\rho_{3}\left(a_{1} \sigma_{2,2}-a_{2} \sigma_{1,2}\right),
\end{aligned}
$$

and $\tilde{s}:=\left(\tilde{s}_{1}, \tilde{s}_{2}, \tilde{s}_{3}\right)^{\top}, \tilde{\xi}:=\left(\rho_{3},-\rho_{2}, \rho_{1}\right)^{\top}$. It can be easily checked that 


$$
\begin{aligned}
& \langle\tilde{\xi}, \tilde{\eta}\rangle^{2}=-\tilde{s}_{3}^{2} \operatorname{det} B, \\
& \|\tilde{\eta}\|^{2}=-\langle B \tilde{s}, \tilde{s}\rangle+\frac{\kappa^{2}}{\delta^{4}}\langle v, v\rangle-2 \mathrm{i} \frac{\kappa}{\delta^{2}}\left(\left(\tilde{s}_{1} a_{1}+\tilde{s}_{2} a_{2}\right) \delta^{2}+\tilde{s}_{3}\left(a_{1} \delta_{3}+a_{2} \delta_{1}\right)\right), \\
& \tilde{s}^{\top} B \tilde{s}=b_{1,1}\left(\tilde{s}_{1}+\frac{b_{1,2} \tilde{s}_{2}+b_{1,3} \tilde{s}_{3}}{b_{1,1}}\right)^{2}+\frac{1}{b_{1,1}}\left[\begin{array}{c}
\tilde{s}_{2} \\
\tilde{s}_{3}
\end{array}\right]^{\top}\left[\begin{array}{ll}
\delta^{2} & \delta_{1} \\
\delta_{1} & \delta_{4}
\end{array}\right]\left[\begin{array}{l}
\tilde{s}_{2} \\
\tilde{s}_{3}
\end{array}\right],
\end{aligned}
$$

where $\delta_{3}:=b_{1,3} b_{2,2}-b_{1,2} b_{2,3}$ and $\delta_{4}:=b_{1,1} b_{3,3}-b_{1,3}^{2}$. Using (4.1), the identities above and (5.8), the partial Fourier transform $\tilde{f}_{2,3}$ can be calculated as follows

$$
\begin{gathered}
\tilde{f}_{2,3}\left(s_{1}, \tilde{s}_{2}, \tilde{s}_{3}\right)=\sqrt{\frac{\left|\tilde{s}_{3}\right| \delta}{2 \pi b_{1,1} \sinh \left(\left|\tilde{s}_{3}\right| \delta\right)}} \exp \left\{-\frac{\kappa}{2(1+\kappa) \delta^{2}}\left(b_{2,2} a_{1}^{2}-2 b_{1,2} a_{1} a_{2}+b_{1,1} a_{2}^{2}\right)\right. \\
-\frac{\kappa}{2(1+\kappa) \delta^{2}} \tilde{s}_{3}^{2} \operatorname{det} B-\frac{1}{2(1+\kappa) b_{1,1}}\left[\begin{array}{c}
\tilde{s}_{2} \\
\tilde{s}_{3}
\end{array}\right]^{\top}\left[\begin{array}{ll}
\delta^{2} & \delta_{1} \\
\delta_{1} & \delta_{4}
\end{array}\right]\left[\begin{array}{c}
\tilde{s}_{2} \\
\tilde{s}_{3}
\end{array}\right]-\frac{1+\kappa}{2 b_{1,1}}\left(\frac{a_{1}}{1+\kappa}-s_{1}\right)^{2} \\
\left.-\frac{b_{1,2} \tilde{s}_{2}+b_{1,3} \tilde{s}_{3}}{b_{1,1}}\left(\frac{a_{1}}{1+\kappa}-s_{1}\right)+\mathrm{i}\left(\tilde{s}_{2} a_{2}+\tilde{s}_{3} a_{3}-\frac{\kappa}{(1+\kappa) \delta^{2}}\left(\tilde{s}_{2} a_{2} \delta^{2}+\tilde{s}_{3}\left(a_{1} \delta_{3}+a_{2} \delta_{1}\right)\right)\right)\right\} .
\end{gathered}
$$

Finally Proposition 4.1 implies that the Fourier transform $\hat{\mu}\left(\pi_{ \pm \lambda}\right)$ is an integral operator on $L^{2}(\mathbb{R})$,

$$
\left[\hat{\mu}\left(\pi_{ \pm \lambda}\right) u\right](x)=\int_{\mathbb{R}} K_{ \pm \lambda}(x, y) u(y) \mathrm{d} y,
$$

where $K_{ \pm \lambda}$ has the form given in Theorem 3.1.

\section{Convolution of Gaussian measures}

The convolution of two probability measures $\mu^{\prime}$ and $\mu^{\prime \prime}$ on $\mathbb{H}$ is defined by

$$
\left(\mu^{\prime} * \mu^{\prime \prime}\right)(A):=\int_{\mathbb{H}} \mu^{\prime \prime}\left(h^{-1} A\right) \mu^{\prime}(\mathrm{d} h),
$$

for all Borel sets $A$ in $\mathbb{H}$.

First we give an explicit formula for the Fourier transform of a convolution of two Gaussian measures on $\mathbb{H}$.

Theorem 6.1. Let $\mu^{\prime}$ and $\mu^{\prime \prime}$ be Gaussian measures on $\mathbb{H}$ with parameters $\left(a^{\prime}, B^{\prime}\right)$ and $\left(a^{\prime \prime}, B^{\prime \prime}\right)$, respectively. Then we have

$$
\begin{aligned}
& \left(\mu^{\prime} * \mu^{\prime \prime}\right)^{\wedge}\left(\chi_{\alpha, \beta}\right)=\exp \left\{\mathrm{i}\left(\left(a_{1}^{\prime}+a_{1}^{\prime \prime}\right) \alpha+\left(a_{2}^{\prime}+a_{2}^{\prime \prime}\right) \beta\right)\right. \\
& \left.-\frac{1}{2}\left(\left(b_{1,1}^{\prime}+b_{1,1}^{\prime \prime}\right) \alpha^{2}+2\left(b_{1,2}^{\prime}+b_{1,2}^{\prime \prime}\right) \alpha \beta+\left(b_{2,2}^{\prime}+b_{2,2}^{\prime \prime}\right) \beta^{2}\right)\right\}, \\
& {\left[\left(\mu^{\prime} * \mu^{\prime \prime}\right)^{\wedge}\left(\pi_{ \pm \lambda}\right) u\right](x)= \begin{cases}L_{ \pm \lambda}(x) u\left(x+\sqrt{\lambda}\left(a_{1}^{\prime}+a_{1}^{\prime \prime}\right)\right) & \text { if } b_{1,1}^{\prime}=b_{1,1}^{\prime \prime}=0, \\
\int_{\mathbb{R}} K_{ \pm \lambda}(x, y) u(y) \mathrm{d} y & \text { otherwise, }\end{cases} }
\end{aligned}
$$

where $L_{ \pm \lambda}(x)$ is given by

$$
\begin{aligned}
\exp & \left\{ \pm \mathrm{i}\left(\lambda\left(a_{3}^{\prime}+a_{3}^{\prime \prime}+\left(a_{1}^{\prime} a_{2}^{\prime}+a_{1}^{\prime \prime} a_{2}^{\prime \prime}\right) / 2\right)+\sqrt{\lambda}\left(a_{2}^{\prime}+a_{2}^{\prime \prime}\right) x+\lambda a_{1}^{\prime} a_{2}^{\prime \prime}\right)\right. \\
& -\frac{\lambda^{2}}{2}\left(b_{3,3}^{\prime}+b_{3,3}^{\prime \prime}+a_{1}^{\prime} b_{2,3}^{\prime}+\left(2 a_{1}^{\prime}+a_{1}^{\prime \prime}\right) b_{2,3}^{\prime \prime}+\left(\left(a_{1}^{\prime}\right)^{2} b_{2,2}^{\prime}+\left(a_{1}^{\prime \prime}\right)^{2} b_{2,2}^{\prime \prime}\right) / 3+a_{1}^{\prime}\left(a_{1}^{\prime}+a_{1}^{\prime \prime}\right) b_{2,2}^{\prime \prime}\right) \\
& \left.-\frac{\lambda^{3 / 2}}{2} x\left(2 b_{2,3}^{\prime}+2 b_{2,3}^{\prime \prime}+a_{1}^{\prime} b_{2,2}^{\prime}+\left(2 a_{1}^{\prime}+a_{1}^{\prime \prime}\right) b_{2,2}^{\prime \prime}\right)-\frac{\lambda}{2} x^{2}\left(b_{2,2}^{\prime}+b_{2,2}^{\prime \prime}\right)\right\},
\end{aligned}
$$


and $K_{ \pm \lambda}(x, y):=C \exp \left\{-\frac{1}{2} \mathbf{z}^{\top} V \mathbf{z}\right\}, \mathbf{z}:=(x, y, 1)^{\top}$, with

$$
C:= \begin{cases}C_{ \pm \lambda}\left(B^{\prime}\right) & \text { if } b_{1,1}^{\prime}>0 \text { and } b_{1,1}^{\prime \prime}=0, \\ C_{ \pm \lambda}\left(B^{\prime \prime}\right) & \text { if } b_{1,1}^{\prime}=0 \text { and } b_{1,1}^{\prime \prime}>0, \\ C_{ \pm \lambda}\left(B^{\prime}\right) C_{ \pm \lambda}\left(B^{\prime \prime}\right) \sqrt{\frac{2 \pi}{d_{2,2}^{\prime}+d_{1,1}^{\prime \prime}}} & \text { if } b_{1,1}^{\prime}>0 \text { and } b_{1,1}^{\prime \prime}>0\end{cases}
$$

(taking the square root with positive real part) and

$$
V:=\left\{\begin{array}{cccc}
D_{ \pm \lambda}\left(a^{\prime}, B^{\prime}\right)+\left[\begin{array}{ccc}
0 & 0 & -\sqrt{\lambda} a_{1}^{\prime \prime} d_{1,2}^{\prime} \\
0 & \lambda b_{2,2}^{\prime \prime} & p_{2,3} \\
-\sqrt{\lambda} a_{1}^{\prime \prime} d_{1,2}^{\prime} & p_{3,2} & p_{3,3}
\end{array}\right] & \text { if } b_{1,1}^{\prime}>0 \text { and } b_{1,1}^{\prime \prime}=0, \\
{\left[\begin{array}{ccc}
\lambda b_{2,2}^{\prime} & 0 & q_{1,3} \\
0 & 0 & \sqrt{\lambda} a_{1}^{\prime} d_{1,2}^{\prime \prime} \\
q_{3,1} & \sqrt{\lambda} a_{1}^{\prime} d_{1,2}^{\prime \prime} & q_{3,3}
\end{array}\right]+D_{ \pm \lambda}\left(a^{\prime \prime}, B^{\prime \prime}\right)} & \text { if } b_{1,1}^{\prime}=0 \text { and } b_{1,1}^{\prime \prime}>0, \\
{\left[\begin{array}{ccc}
d_{1,1}^{\prime} & 0 & d_{1,3}^{\prime} \\
0 & d_{2,2}^{\prime \prime} & d_{2,3}^{\prime \prime} \\
d_{3,1}^{\prime} & d_{3,2}^{\prime \prime} & d_{3,3}^{\prime}+d_{3,3}^{\prime \prime}
\end{array}\right]-\frac{U U^{\top}}{d_{2,2}^{\prime}+d_{1,1}^{\prime \prime}}} & \text { if } b_{1,1}^{\prime}>0 \text { and } b_{1,1}^{\prime \prime}>0,
\end{array}\right.
$$

where $d_{j, k}^{\prime}:=d_{j, k}^{ \pm \lambda}\left(a^{\prime}, B^{\prime}\right), d_{j, k}^{\prime \prime}:=d_{j, k}^{ \pm \lambda}\left(a^{\prime \prime}, B^{\prime \prime}\right)$ for $1 \leqslant j, k \leqslant 3$ and

$$
\begin{aligned}
& U:=\left(d_{1,2}^{\prime}, d_{2,1}^{\prime \prime}, d_{3,2}^{\prime}+d_{3,1}^{\prime \prime}\right)^{\top}, \\
& p_{2,3}:=p_{3,2}:=-\sqrt{\lambda} a_{1}^{\prime \prime} d_{2,2}^{\prime}+\lambda^{3 / 2}\left(2 b_{2,3}^{\prime \prime}-a_{1}^{\prime \prime} b_{2,2}^{\prime \prime}\right) / 2 \mp \mathrm{i} \sqrt{\lambda} a_{2}^{\prime \prime}, \\
& p_{3,3}:=-\sqrt{\lambda} a_{1}^{\prime \prime}\left(d_{2,3}^{\prime}+d_{3,2}^{\prime}\right)+\lambda\left(a_{1}^{\prime \prime}\right)^{2} d_{2,2}^{\prime}+\lambda^{2}\left(b_{3,3}^{\prime \prime}-a_{1}^{\prime \prime} b_{2,3}^{\prime \prime}+\left(a_{1}^{\prime \prime}\right)^{2} b_{2,2}^{\prime \prime} / 3\right) \mp \mathrm{i} \lambda\left(2 a_{3}^{\prime \prime}-a_{1}^{\prime \prime} a_{2}^{\prime \prime}\right), \\
& q_{1,3}:=q_{3,1}:=\sqrt{\lambda} a_{1}^{\prime} d_{1,1}^{\prime \prime}+\lambda^{3 / 2}\left(a_{1}^{\prime} b_{2,2}^{\prime}+2 b_{2,3}^{\prime}\right) / 2 \mp \mathrm{i} \sqrt{\lambda} a_{2}^{\prime}, \\
& q_{3,3}:=\sqrt{\lambda} a_{1}^{\prime}\left(d_{1,3}^{\prime \prime}+d_{3,1}^{\prime \prime}\right)+\lambda\left(a_{1}^{\prime}\right)^{2} d_{1,1}^{\prime \prime}+\lambda^{2}\left(b_{3,3}^{\prime}+a_{1}^{\prime} b_{2,3}^{\prime}+\left(a_{1}^{\prime}\right)^{2} b_{2,2}^{\prime} / 3\right) \mp \mathrm{i} \lambda\left(2 a_{3}^{\prime}+a_{1}^{\prime} a_{2}^{\prime}\right) .
\end{aligned}
$$

Proof. If $b_{1,1}^{\prime}>0$ and $b_{1,1}^{\prime \prime}>0$ then the assertion can be proved as in Pap [10, Theorem 7.2]. If $b_{1,1}^{\prime}>0$ and $b_{1,1}^{\prime \prime}=0$ then by Theorem 3.1

$$
\left[\widehat{\mu^{\prime}}\left(\pi_{ \pm \lambda}\right) u\right](x)=\int_{\mathbb{R}} K_{ \pm \lambda}^{\prime}(x, y) u(y) \mathrm{d} y
$$

with

$$
K_{ \pm \lambda}^{\prime}(x, y):=C_{ \pm \lambda}\left(B^{\prime}\right) \exp \left\{-\frac{1}{2} \mathbf{z}^{\top} D_{ \pm \lambda}\left(a^{\prime}, B^{\prime}\right) \mathbf{z}\right\}, \quad \mathbf{z}=(x, y, 1)^{\top}
$$

and

$$
\begin{aligned}
{\left[\widehat{\mu^{\prime \prime}}\left(\pi_{ \pm \lambda}\right) u\right](y)=} & \exp \left\{ \pm \frac{\mathrm{i} \sqrt{\lambda}}{2}\left(\sqrt{\lambda}\left(2 a_{3}^{\prime \prime}+a_{1}^{\prime \prime} a_{2}^{\prime \prime}\right)+2 a_{2}^{\prime \prime} y\right)-\frac{\lambda^{2}}{6}\left(3 b_{3,3}^{\prime \prime}+3 a_{1}^{\prime \prime} b_{2,3}^{\prime \prime}+\left(a_{1}^{\prime \prime}\right)^{2} b_{2,2}^{\prime \prime}\right)\right. \\
& \left.-\frac{\lambda^{3 / 2}}{2}\left(2 b_{2,3}^{\prime \prime}+a_{1}^{\prime \prime} b_{2,2}^{\prime \prime}\right) y-\frac{\lambda}{2} b_{2,2}^{\prime \prime} y^{2}\right\} u\left(y+\sqrt{\lambda} a_{1}^{\prime \prime}\right) .
\end{aligned}
$$

Clearly we have

$$
\left[\left(\mu^{\prime} * \mu^{\prime \prime}\right)^{\wedge}\left(\pi_{ \pm \lambda}\right) u\right](x)=\left[\widehat{\mu^{\prime}}\left(\pi_{ \pm \lambda}\right) \widehat{\mu^{\prime \prime}}\left(\pi_{ \pm \lambda}\right) u\right](x)=\int_{\mathbb{R}} K_{ \pm \lambda}^{\prime}(x, y)\left[\widehat{\mu^{\prime \prime}}\left(\pi_{ \pm \lambda}\right) u\right](y) \mathrm{d} y .
$$

Using the formulas for $\widehat{\mu^{\prime}}\left(\pi_{ \pm \lambda}\right)$ and $\widehat{\mu^{\prime \prime}}\left(\pi_{ \pm \lambda}\right)$ an easy calculation yields that $K_{ \pm \lambda}$ has the form given in the theorem. The other cases $b_{1,1}^{\prime}=0, b_{1,1}^{\prime \prime}>0$ and $b_{1,1}^{\prime}=b_{1,1}^{\prime \prime}=0$ can be handled in the same way.

We need two lemmas concerning the parameters of a Gaussian measure on $\mathbb{H}$. 
Lemma 6.1. Let us consider a Gaussian semigroup $\left(\mu_{t}\right)_{t} \geqslant 0$ such that $\mu_{1}$ is a Gaussian measure on $\mathbb{H}$ with parameters $(a, B)$. Then we have

$$
a_{i}=\mathrm{E} Z_{i}, \quad i=1,2,3, \quad b_{i, j}=\operatorname{Cov}\left(Z_{i}, Z_{j}\right) \quad \text { if }(i, j) \neq(3,3),
$$

and

$$
\begin{aligned}
b_{3,3}= & \operatorname{Var} Z_{3}-\frac{1}{4}\left(\operatorname{Var} Z_{1} \operatorname{Var} Z_{2}-\operatorname{Cov}\left(Z_{1}, Z_{2}\right)^{2}\right) \\
& -\frac{1}{12}\left(\operatorname{Var} Z_{2}\left(E Z_{1}\right)^{2}-2 \operatorname{Cov}\left(Z_{1}, Z_{2}\right) \mathrm{E} Z_{1} \mathrm{E} Z_{2}+\operatorname{Var} Z_{1}\left(E Z_{2}\right)^{2}\right),
\end{aligned}
$$

where the distribution of the random vector $\left(Z_{1}, Z_{2}, Z_{3}\right)$ with values in $\mathbb{R}^{3}$ is $\mu_{1}$.

Proof. Let $Z(t):=\left(Z_{1}(t), Z_{2}(t), Z_{3}(t)\right), t \geqslant 0$ be given as in Section 2 . Taking the expectation of $Z(1)$ yields that $\mathrm{E}\left(Z_{i}(1)\right)=a_{i}, i=1,2,3$. Using again the definition of $Z(1)$ and the fact that $B=\Sigma \cdot \Sigma^{\top}$ we get

$$
\operatorname{Var}\left(Z_{1}(1)\right)=\sum_{k=1}^{d} \sum_{\ell=1}^{d} \sigma_{1, k} \sigma_{1, \ell} \mathrm{E}\left(W_{k}(1) W_{\ell}(1)\right)=\sum_{k=1}^{d} \sigma_{1, k}^{2}=b_{1,1} .
$$

Similar arguments show $\operatorname{Var}\left(Z_{2}(1)\right)=b_{2,2}$ and $\operatorname{Cov}\left(Z_{1}(1), Z_{2}(1)\right)=b_{1,2}$. We also obtain

$$
\begin{aligned}
\operatorname{Cov}\left(Z_{1}(1), Z_{3}(1)\right)= & \mathrm{E}\left[\sum_{i=1}^{d} \sigma_{1, i} W_{i}(1)\left(\sum_{k=1}^{d} \sigma_{3, k} W_{k}(1)+\sum_{k=1}^{d}\left(a_{2} \sigma_{1, k}-a_{1} \sigma_{2, k}\right) W_{k}^{*}(1)\right)\right. \\
& \left.+\sum_{i=1}^{d} \sigma_{1, i} W_{i}(1) \sum_{1 \leqslant k<\ell \leqslant d}\left(\sigma_{1, k} \sigma_{2, \ell}-\sigma_{1, \ell} \sigma_{2, k}\right) W_{k, \ell}(1)\right],
\end{aligned}
$$

which implies that

$$
\begin{aligned}
\operatorname{Cov}\left(Z_{1}(1), Z_{3}(1)\right)= & \sum_{k=1}^{d} \sigma_{1, k} \sigma_{3, k}+\sum_{i=1}^{d} \sum_{k=1}^{d} \sigma_{1, i}\left(a_{2} \sigma_{1, k}-a_{1} \sigma_{2, k}\right) \mathrm{E}\left(W_{i}(1) W_{k}^{*}(1)\right) \\
& +\sum_{i=1}^{d} \sum_{1 \leqslant k<\ell \leqslant d} \sigma_{1, i}\left(\sigma_{1, k} \sigma_{2, \ell}-\sigma_{1, \ell} \sigma_{2, k}\right) \mathrm{E}\left(W_{i}(1) W_{k, \ell}(1)\right)=b_{1,3},
\end{aligned}
$$

since $W_{i}(1), 1 \leqslant i \leqslant d$, are independent of each other and

$$
\mathrm{E}\left(W_{i}(1) W_{k}^{*}(1)\right)=\mathrm{E}\left(W_{i}(1) W_{k, \ell}(1)\right)=0, \quad 1 \leqslant i \leqslant d, 1 \leqslant k<\ell \leqslant d .
$$

Indeed,

$$
\begin{aligned}
\mathrm{E}\left(W_{i}(1) W_{k}^{*}(1)\right)=\frac{1}{2} \lim _{n \rightarrow \infty} \mathrm{E} & {\left[W_{i}(1) \sum_{j=1}^{n}\left(W_{k}\left(s_{j-1}^{(n)}\right)\left(s_{j}^{(n)}-s_{j-1}^{(n)}\right)-s_{j-1}^{(n)}\left(W_{k}\left(s_{j}^{(n)}\right)-W_{k}\left(s_{j-1}^{(n)}\right)\right)\right)\right], } \\
\mathrm{E}\left(W_{i}(1) W_{k, \ell}(1)\right)=\frac{1}{2} \lim _{n \rightarrow \infty} \mathrm{E} & {\left[W _ { i } ( 1 ) \sum _ { j = 1 } ^ { n } \left(W_{k}\left(s_{j-1}^{(n)}\right)\left(W_{\ell}\left(s_{j}^{(n)}\right)-W_{\ell}\left(s_{j-1}^{(n)}\right)\right)\right.\right.} \\
& \left.\left.-W_{\ell}\left(s_{j-1}^{(n)}\right)\left(W_{k}\left(s_{j}^{(n)}\right)-W_{k}\left(s_{j-1}^{(n)}\right)\right)\right)\right]
\end{aligned}
$$

for all $1 \leqslant i \leqslant d, 1 \leqslant k<\ell \leqslant d$, where $\left\{s_{j}^{(n)}: j=0, \ldots, n\right\}$ denotes a partition of the interval [0,1] such that $\max _{1 \leqslant j \leqslant n}\left(s_{j}^{(n)}-s_{j-1}^{(n)}\right)$ tends to 0 as $n$ goes to infinity. We can obtain $\operatorname{Cov}\left(Z_{2}(1), Z_{3}(1)\right)=b_{2,3}$ in the same way. Using again the form of $Z(t),(6.1)$ and the facts that 


$$
\begin{aligned}
& \operatorname{Cov}\left(W_{i, j}(1), W_{k, \ell}(1)\right)=0 \text { for all } 1 \leqslant i<j \leqslant d, 1 \leqslant k<\ell \leqslant d,(i, j) \neq(k, \ell), \\
& \operatorname{Cov}\left(W_{k}^{*}(1), W_{\ell}^{*}(1)\right)=0 \text { for all } 1 \leqslant k, \ell \leqslant d, k \neq \ell,
\end{aligned}
$$

we get

$$
\operatorname{Var}\left(Z_{3}(1)\right)=\sum_{k=1}^{d} \sigma_{3, k}^{2}+\sum_{k=1}^{d}\left(a_{2} \sigma_{1, k}-a_{1} \sigma_{2, k}\right)^{2} \operatorname{Var}\left(W_{k}^{*}(1)\right)+\sum_{1 \leqslant k<\ell \leqslant d}\left(\sigma_{1, k} \sigma_{2, \ell}-\sigma_{1, \ell} \sigma_{2, k}\right)^{2} \operatorname{Var}\left(W_{k, \ell}(1)\right) .
$$

Lévy proved that the (Euclidean) Fourier transform of $W_{k, \ell}(1), 1 \leqslant k<\ell \leqslant d$ (i.e., the characteristic function of $W_{k, \ell}$ ) is

$$
\mathrm{E}\left(\mathrm{e}^{\mathrm{i} t W_{k, \ell}(1)}\right)=\frac{1}{\cosh (t / 2)}, \quad 1 \leqslant k<\ell \leqslant d,
$$

for all $t \in \mathbb{R}$ (this follows also from Proposition 5.1), so

$$
\operatorname{Var}\left(W_{k, \ell}(1)\right)=-\left.\frac{\mathrm{d}^{2}}{\mathrm{~d} t^{2}}\left(\frac{1}{\cosh (t / 2)}\right)\right|_{t=0}=\frac{1}{4}, \quad 1 \leqslant k<\ell \leqslant d .
$$

Clearly $W_{k}^{*}$ has a normal distribution with zero mean and with variance $\operatorname{Var}\left(W_{k}^{*}(1)\right)=\frac{1}{12}, 1 \leqslant k \leqslant d$. Using (5.11) we have

$$
\operatorname{Var}\left(Z_{3}(1)\right)=b_{3,3}+\frac{1}{4}\left(b_{1,1} b_{2,2}-b_{1,2}^{2}\right)+\frac{1}{12}\left(a_{1}^{2} b_{2,2}-2 a_{1} a_{2} b_{1,2}+a_{2}^{2} b_{1,1}\right) .
$$

Hence the assertion.

Lemma 6.2. Let $\mu^{\prime}$ and $\mu^{\prime \prime}$ be Gaussian measures on $\mathbb{H}$ with parameters $\left(a^{\prime}, B^{\prime}\right)$ and $\left(a^{\prime \prime}, B^{\prime \prime}\right)$, respectively. If the convolution $\mu^{\prime} * \mu^{\prime \prime}$ is a Gaussian measure on $\mathbb{H}$ with parameters $(a, B)$ then we have

$$
\begin{aligned}
a_{1}= & a_{1}^{\prime}+a_{1}^{\prime \prime}, \quad a_{2}=a_{2}^{\prime}+a_{2}^{\prime \prime}, \quad a_{3}=a_{3}^{\prime}+a_{3}^{\prime \prime}+\frac{1}{2}\left(a_{1}^{\prime} a_{2}^{\prime \prime}-a_{2}^{\prime} a_{1}^{\prime \prime}\right), \\
b_{1,1}= & b_{1,1}^{\prime}+b_{1,1}^{\prime \prime}, \quad b_{1,2}=b_{1,2}^{\prime}+b_{1,2}^{\prime \prime}, \quad b_{2,2}=b_{2,2}^{\prime}+b_{2,2}^{\prime \prime}, \\
b_{1,3}= & b_{1,3}^{\prime}+b_{1,3}^{\prime \prime}+\frac{1}{2}\left(a_{2}^{\prime \prime} b_{1,1}^{\prime}-a_{1}^{\prime \prime} b_{1,2}^{\prime}+a_{1}^{\prime} b_{1,2}^{\prime \prime}-a_{2}^{\prime} b_{1,1}^{\prime \prime}\right), \\
b_{2,3}= & b_{2,3}^{\prime}+b_{2,3}^{\prime \prime}+\frac{1}{2}\left(a_{2}^{\prime \prime} b_{1,2}^{\prime}-a_{1}^{\prime \prime} b_{2,2}^{\prime}+a_{1}^{\prime} b_{2,2}^{\prime \prime}-a_{2}^{\prime} b_{1,2}^{\prime \prime}\right), \\
b_{3,3}= & b_{3,3}^{\prime}+b_{3,3}^{\prime \prime}+a_{2}^{\prime \prime} b_{1,3}^{\prime}-a_{1}^{\prime \prime} b_{2,3}^{\prime}+a_{1}^{\prime} b_{2,3}^{\prime \prime}-a_{2}^{\prime} b_{1,3}^{\prime \prime} \\
& +\frac{1}{6}\left(-a_{1}^{\prime} a_{1}^{\prime \prime} b_{2,2}^{\prime}+\left(a_{1}^{\prime \prime}\right)^{2} b_{2,2}^{\prime}+\left(a_{1}^{\prime}\right)^{2} b_{2,2}^{\prime \prime}-a_{1}^{\prime} a_{2}^{\prime \prime} b_{2,2}^{\prime \prime}+a_{1}^{\prime} a_{2}^{\prime \prime} b_{1,2}^{\prime}+a_{1}^{\prime \prime} a_{2}^{\prime} b_{1,2}^{\prime}-2 a_{1}^{\prime \prime} a_{2}^{\prime \prime} b_{1,2}^{\prime}\right. \\
& \left.\quad-2 a_{1}^{\prime} a_{2}^{\prime} b_{1,2}^{\prime \prime}+a_{1}^{\prime} a_{2}^{\prime \prime} b_{1,2}^{\prime \prime}+a_{1}^{\prime \prime} a_{2}^{\prime} b_{1,2}^{\prime \prime}-a_{2}^{\prime} a_{2}^{\prime \prime} b_{1,1}^{\prime}+\left(a_{2}^{\prime \prime}\right)^{2} b_{1,1}^{\prime}+\left(a_{2}^{\prime}\right)^{2} b_{1,1}^{\prime \prime}-a_{2}^{\prime} a_{2}^{\prime \prime} b_{1,1}^{\prime \prime}\right) .
\end{aligned}
$$

Proof. Let $Z^{\prime}=\left(Z_{1}^{\prime}, Z_{2}^{\prime}, Z_{3}^{\prime}\right)^{\top}$ and $Z^{\prime \prime}=\left(Z_{1}^{\prime \prime}, Z_{2}^{\prime \prime}, Z_{3}^{\prime \prime}\right)^{\top}$ be independent random variables with values in $\mathbb{R}^{3}$ such that the distribution of $Z^{\prime}$ is $\mu^{\prime}$ and the distribution of $Z^{\prime \prime}$ is $\mu^{\prime \prime}$, respectively. Then the convolution $\mu^{\prime} * \mu^{\prime \prime}$ is the distribution of the random variable

$$
\left(Z_{1}^{\prime}+Z_{1}^{\prime \prime}, Z_{2}^{\prime}+Z_{2}^{\prime \prime}, Z_{3}^{\prime}+Z_{3}^{\prime \prime}+\frac{1}{2}\left(Z_{1}^{\prime} Z_{2}^{\prime \prime}-Z_{1}^{\prime \prime} Z_{2}^{\prime}\right)\right)=:\left(Z_{1}, Z_{2}, Z_{3}\right)
$$

Using Lemma 6.1 we get

$$
\begin{aligned}
& a_{1}=\mathrm{E} Z_{1}=\mathrm{E} Z_{1}^{\prime}+\mathrm{E} Z_{1}^{\prime \prime}=a_{1}^{\prime}+a_{1}^{\prime \prime}, \\
& a_{2}=\mathrm{E} Z_{2}=\mathrm{E} Z_{2}^{\prime}+\mathrm{E} Z_{2}^{\prime \prime}=a_{2}^{\prime}+a_{2}^{\prime \prime}, \\
& a_{3}=\mathrm{E} Z_{3}=\mathrm{E} Z_{3}^{\prime}+\mathrm{E} Z_{3}^{\prime \prime}+\frac{1}{2}\left(\mathrm{E}_{1}^{\prime} \mathrm{E} Z_{2}^{\prime \prime}-\mathrm{E} Z_{1}^{\prime \prime} \mathrm{E} Z_{2}^{\prime}\right)=a_{3}^{\prime}+a_{3}^{\prime \prime}+\frac{1}{2}\left(a_{1}^{\prime} a_{2}^{\prime \prime}-a_{2}^{\prime} a_{1}^{\prime \prime}\right),
\end{aligned}
$$

since $Z^{\prime}$ and $Z^{\prime \prime}$ are independent of each other. Similar arguments show that 


$$
\begin{aligned}
& b_{1,1}=\operatorname{Var} Z_{1}=\operatorname{Var} Z_{1}^{\prime}+\operatorname{Var} Z_{1}^{\prime \prime}=b_{1,1}^{\prime}+b_{1,1}^{\prime \prime}, \\
& b_{2,2}=\operatorname{Var} Z_{2}=\operatorname{Var} Z_{2}^{\prime}+\operatorname{Var} Z_{2}^{\prime \prime}=b_{2,2}^{\prime}+b_{2,2}^{\prime \prime}, \\
& b_{1,2}=\operatorname{Cov}\left(Z_{1}, Z_{2}\right)=b_{1,2}^{\prime}+b_{1,2}^{\prime \prime} .
\end{aligned}
$$

We also have

$$
\begin{aligned}
b_{1,3}=\operatorname{Cov}\left(Z_{1}, Z_{3}\right)= & \operatorname{Cov}\left(Z_{1}^{\prime}, Z_{3}^{\prime}\right)+\operatorname{Cov}\left(Z_{1}^{\prime \prime}, Z_{3}^{\prime \prime}\right) \\
& +\frac{1}{2}\left(\operatorname{Cov}\left(Z_{1}^{\prime}, Z_{1}^{\prime} Z_{2}^{\prime \prime}\right)-\operatorname{Cov}\left(Z_{1}^{\prime}, Z_{2}^{\prime} Z_{1}^{\prime \prime}\right)+\operatorname{Cov}\left(Z_{1}^{\prime \prime}, Z_{1}^{\prime} Z_{2}^{\prime \prime}\right)-\operatorname{Cov}\left(Z_{1}^{\prime \prime}, Z_{1}^{\prime \prime} Z_{2}^{\prime}\right)\right) .
\end{aligned}
$$

Using this and Lemma 6.1 the validity of the formula for $b_{1,3}$ can be easily checked. For example we have

$$
\operatorname{Cov}\left(Z_{1}^{\prime}, Z_{1}^{\prime} Z_{2}^{\prime \prime}\right)=\mathrm{E}\left(\left(Z_{1}^{\prime}\right)^{2} Z_{2}^{\prime \prime}\right)-\mathrm{E} Z_{1}^{\prime} \mathrm{E}\left(Z_{1}^{\prime} Z_{2}^{\prime \prime}\right)=\left(b_{1,1}^{\prime}+\left(a_{1}^{\prime}\right)^{2}\right) a_{2}^{\prime \prime}-\left(a_{1}^{\prime}\right)^{2} a_{2}^{\prime \prime}=a_{2}^{\prime \prime} b_{1,1}^{\prime} .
$$

The validity of the formula for $b_{2,3}$ can be proved in the same way. Lemma 6.1 implies that

$$
\begin{gathered}
\operatorname{Var} Z_{3}=b_{3,3}+\frac{1}{4}\left(b_{1,1} b_{2,2}-b_{1,2}^{2}\right)+\frac{1}{12}\left(a_{1}^{2} b_{2,2}-2 a_{1} a_{2} b_{1,2}+a_{2}^{2} b_{1,1}\right)=\operatorname{Cov}\left(Z_{3}, Z_{3}\right) \\
=\operatorname{Cov}\left(Z_{3}^{\prime}, Z_{3}^{\prime}\right)+\operatorname{Cov}\left(Z_{3}^{\prime \prime}, Z_{3}^{\prime \prime}\right)+\operatorname{Cov}\left(Z_{3}^{\prime}, Z_{1}^{\prime} Z_{2}^{\prime \prime}\right)-\operatorname{Cov}\left(Z_{3}^{\prime}, Z_{1}^{\prime \prime} Z_{2}^{\prime}\right)+\operatorname{Cov}\left(Z_{3}^{\prime \prime}, Z_{1}^{\prime} Z_{2}^{\prime \prime}\right) \\
-\operatorname{Cov}\left(Z_{3}^{\prime \prime}, Z_{1}^{\prime \prime} Z_{2}^{\prime}\right)+\frac{1}{4}\left(\operatorname{Cov}\left(Z_{1}^{\prime} Z_{2}^{\prime \prime}, Z_{1}^{\prime} Z_{2}^{\prime \prime}\right)-\operatorname{Cov}\left(Z_{1}^{\prime} Z_{2}^{\prime \prime}, Z_{1}^{\prime \prime} Z_{2}^{\prime}\right)\right. \\
\left.-\operatorname{Cov}\left(Z_{1}^{\prime \prime} Z_{2}^{\prime}, Z_{1}^{\prime} Z_{2}^{\prime \prime}\right)+\operatorname{Cov}\left(Z_{1}^{\prime \prime} Z_{2}^{\prime}, Z_{1}^{\prime \prime} Z_{2}^{\prime}\right)\right) .
\end{gathered}
$$

Using again Lemma 6.1 and substituting the formulas for $b_{1,1}, b_{1,2}, b_{2,2}, a_{1}$ and $a_{2}$ into the formula above, an easy calculation shows the validity of the formula for $b_{3,3}$.

Our aim is to give necessary and sufficient conditions for a convolution of two Gaussian measures to be a Gaussian measure. Using the fact that the Fourier transform is injective (i.e., if $\mu$ and $v$ are probability measures on $\mathbb{H}$ such that $\hat{\mu}\left(\chi_{\alpha, \beta}\right)=\widehat{v}\left(\chi_{\alpha, \beta}\right)$ for all $\alpha, \beta \in \mathbb{R}$ and $\hat{\mu}\left(\pi_{ \pm \lambda}\right)=\widehat{v}\left(\pi_{ \pm \lambda}\right)$ for all $\lambda>0$ then $\left.\mu=v\right)$, our task can be fulfilled in the following way. We take the Fourier transform of the convolution of two Gaussian measures $\mu^{\prime}$ and $\mu^{\prime \prime}$ with parameters $\left(a^{\prime}, B^{\prime}\right)$ and $\left(a^{\prime \prime}, B^{\prime \prime}\right)$ at all one-dimensional and at all Schrödinger representations and then we search for necessary and sufficient conditions under which this Fourier transform has the form given in Theorem 3.1. First we sketch our approach to obtain necessary conditions. By Theorem 6.1, $\left(\mu^{\prime} * \mu^{\prime \prime}\right)^{\wedge}\left(\pi_{ \pm \lambda}\right)$ is an integral operator for $b_{1,1}^{\prime}+b_{1,1}^{\prime \prime}>0$, and it is a product of certain shift and multiplication operators for $b_{1,1}^{\prime}+b_{1,1}^{\prime \prime}=0$. If the convolution $\mu^{\prime} * \mu^{\prime \prime}$ is a Gaussian measure with parameters $(a, B)$ then, by Theorem 3.1, $\left(\mu^{\prime} * \mu^{\prime \prime}\right)^{\wedge}\left(\pi_{ \pm \lambda}\right)$ is an integral operator for $b_{1,1}>0$, and it is a product of certain shift and multiplication operators for $b_{1,1}=0$. By Lemma 6.2, we have $b_{1,1}=b_{1,1}^{\prime}+b_{1,1}^{\prime \prime}$, hence $b_{1,1}=0$ if and only if $b_{1,1}^{\prime}+b_{1,1}^{\prime \prime}=0$. If $\left(\mu^{\prime} * \mu^{\prime \prime}\right)^{\wedge}\left(\pi_{ \pm \lambda}\right)$ is an integral operator then it is uniquely determined by its kernel function $K_{ \pm \lambda}$, hence, by Theorems 3.1 and $6.1, d_{j, k}^{ \pm \lambda}=v_{j, k}^{ \pm \lambda}$ for all $1 \leqslant j, k \leqslant 3$ with $(j, k) \neq(3,3)$ and for all $\lambda>0$, where $d_{j, k}^{ \pm \lambda}:=d_{j, k}^{ \pm \lambda}(a, B), 1 \leqslant j, k \leqslant 3$, and $V=:\left(v_{j, k}^{ \pm \lambda}\right)_{1 \leqslant j, k \leqslant 3}$ are defined in Theorem 3.1 and Theorem 6.1, respectively. (The quantities $d_{3,3}^{ \pm \lambda}, C_{ \pm \lambda}(B)$ from Theorem 3.1 and $v_{3,3}^{ \pm \lambda}, C$ from Theorem 6.1 also satisfy an equation, but we will not use it.) We derive necessary conditions from the above equations and prove that they are also sufficient. This train of thoughts will be used in the proof of Proposition 6.1 and Theorem 6.2.

Remark 6.1. By Lemma 4.2, it can be easily checked that a Gaussian measure $\mu$ admits parameters $(a, B)$ with $b_{j, k}=0$ for $1 \leqslant j, k \leqslant 3$ with $(j, k) \neq(3,3)$ and $a_{1}=a_{2}=0$ if and only if the support of $\mu$ is contained in the center of $\mathbb{H}$.

Now we can derive a special case of Theorem 6.2 which will be used in the proof of Theorem 6.2.

Proposition 6.1. If $\mu^{\prime \prime}$ is a Gaussian measure on $\mathbb{H}$ such that the support of $\mu^{\prime \prime}$ is contained in the center of $\mathbb{H}$ then for all Gaussian measures $\mu^{\prime}$, the convolutions $\mu^{\prime} * \mu^{\prime \prime}$ and $\mu^{\prime \prime} * \mu^{\prime}$ are Gaussian measures with parameters $\left(a^{\prime}+a^{\prime \prime}, B^{\prime}+B^{\prime \prime}\right)$, and $\mu^{\prime} * \mu^{\prime \prime}=\mu^{\prime \prime} * \mu^{\prime}$. 
Proof. Let $\mu$ be a Gaussian measure with parameters $\left(a^{\prime}+a^{\prime \prime}, B^{\prime}+B^{\prime \prime}\right)$. By the injectivity of the Fourier transform, in order to prove that $\mu^{\prime} * \mu^{\prime \prime}=\mu$ is valid, it is sufficient to show that $\left(\mu^{\prime} * \mu^{\prime \prime}\right)^{\prime}\left(\chi_{\alpha, \beta}\right)=\hat{\mu}\left(\chi_{\alpha, \beta}\right)$ for all $\alpha, \beta>0$ and $\left(\mu^{\prime} * \mu^{\prime \prime}\right)^{\wedge}\left(\pi_{ \pm \lambda}\right)=\hat{\mu}\left(\pi_{ \pm \lambda}\right)$ for all $\lambda>0$. Theorem 6.1 implies that $\left(\mu^{\prime} * \mu^{\prime \prime}\right)^{\wedge}\left(\chi_{\alpha, \beta}\right)=\hat{\mu}\left(\chi_{\alpha, \beta}\right)$ is valid for all one-dimensional representations $\chi_{\alpha, \beta}, \alpha, \beta \in \mathbb{R}$. Suppose that $b_{1,1}^{\prime} \neq 0$ and $b_{1,1}^{\prime} b_{2,2}^{\prime}-\left(b_{1,2}^{\prime}\right)^{2} \neq 0$. By Theorem 6.1, to prove $\left(\mu^{\prime} * \mu^{\prime \prime}\right)^{\wedge}\left(\pi_{ \pm \lambda}\right)=\hat{\mu}\left(\pi_{ \pm \lambda}\right)$ for all $\lambda>0$ it is sufficient to show that

$$
D_{ \pm \lambda}\left(a^{\prime}, B^{\prime}\right)+\left[\begin{array}{ccc}
0 & 0 & 0 \\
0 & 0 & 0 \\
0 & 0 & \lambda^{2} b_{3,3}^{\prime \prime} \mp 2 \mathrm{i} \lambda a_{3}^{\prime \prime}
\end{array}\right]=D_{ \pm \lambda}\left(a^{\prime}+a^{\prime \prime}, B^{\prime}+B^{\prime \prime}\right)
$$

for all $\lambda>0$. Since $b_{j, k}^{\prime \prime}=0$ for $1 \leqslant j, k \leqslant 3$ with $(j, k) \neq(3,3)$, we have $d_{j, k}^{ \pm \lambda}\left(a^{\prime}+a^{\prime \prime}, B^{\prime}+B^{\prime \prime}\right)=d_{j, k}^{ \pm \lambda}\left(a^{\prime}, B^{\prime}\right)$ for $1 \leqslant j, k \leqslant 3$ with $(j, k) \neq(3,3)$. So we have to check only that

$$
d_{3,3}^{ \pm \lambda}\left(a^{\prime}, B^{\prime}\right)+\lambda^{2} b_{3,3}^{\prime \prime} \mp 2 \mathrm{i} \lambda a_{3}^{\prime \prime}=d_{3,3}^{ \pm \lambda}\left(a^{\prime}+a^{\prime \prime}, B^{\prime}+B^{\prime \prime}\right)
$$

for all $\lambda>0$. Theorem 3.1 implies this. The case $b_{1,1}^{\prime} \neq 0, b_{1,1}^{\prime} b_{2,2}^{\prime}-\left(b_{1,2}^{\prime}\right)^{2}=0$ can be proved similarly. Suppose that $b_{1,1}^{\prime}=b_{1,1}^{\prime \prime}=0$. Using again Theorem 3.1, we have

$$
\begin{aligned}
& {\left[\widehat{\mu^{\prime \prime}}\left(\pi_{ \pm \lambda}\right) u\right](x)=\exp \{}\left. \pm \mathrm{i} \lambda a_{3}^{\prime \prime}-\frac{\lambda^{2}}{2} b_{3,3}^{\prime \prime}\right\} u(x), \\
& {\left[\widehat{\mu^{\prime}}\left(\pi_{ \pm \lambda}\right) u\right](x)=\exp \left\{ \pm \frac{\mathrm{i} \sqrt{\lambda}}{2}\left(\sqrt{\lambda}\left(2 a_{3}^{\prime}+a_{1}^{\prime} a_{2}^{\prime}\right)+2 a_{2}^{\prime} x\right)-\frac{\lambda^{2}}{6}\left(3 b_{3,3}^{\prime}+3 a_{1}^{\prime} b_{2,3}^{\prime}+\left(a_{1}^{\prime}\right)^{2} b_{2,2}^{\prime}\right)\right.} \\
&\left.-\frac{\lambda^{3 / 2}}{2}\left(2 b_{2,3}^{\prime}+a_{1}^{\prime} b_{2,2}^{\prime}\right) x-\frac{\lambda}{2} b_{2,2}^{\prime} x^{2}\right\} u\left(x+\sqrt{\lambda} a_{1}^{\prime}\right) .
\end{aligned}
$$

Theorem 3.1 implies that $\left[\hat{\mu}\left(\pi_{ \pm \lambda}\right) u\right](x)=\left[\left(\mu^{\prime} * \mu^{\prime \prime}\right)^{\wedge}\left(\pi_{ \pm \lambda}\right) u\right](x)$ for all $\lambda>0, u \in L^{2}(\mathbb{R})$ and $x \in \mathbb{R}$. Hence the assertion.

Now we give necessary and sufficient conditions under which the convolution of two Gaussian measures is a Gaussian measure.

Theorem 6.2. Let $\mu^{\prime}$ and $\mu^{\prime \prime}$ be Gaussian measures on $\mathbb{H}$ with parameters $a^{\prime}=\left(a_{i}^{\prime}\right)_{1 \leqslant i \leqslant 3}, B^{\prime}=\left(b_{j, k}^{\prime}\right)_{1 \leqslant j, k \leqslant 3}$ and $a^{\prime \prime}=\left(a_{i}^{\prime \prime}\right)_{1 \leqslant i \leqslant 3}, B^{\prime \prime}=\left(b_{j, k}^{\prime \prime}\right)_{1 \leqslant j, k \leqslant 3}$, respectively. Then the convolution $\mu^{\prime} * \mu^{\prime \prime}$ is a Gaussian measure on $\mathbb{H}$ if and only if one of the following conditions hold:

$(\widetilde{\mathrm{C}} 1) b_{1,1}^{\prime}>0, \delta^{\prime}>0, b_{1,1}^{\prime \prime}>0, \delta^{\prime \prime}>0$, and there exists $\varrho>0$ such that $b_{j, k}^{\prime \prime}=\varrho b_{j, k}^{\prime}$ for $1 \leqslant j, k \leqslant 3$ with $(j, k) \neq$ $(3,3)$ and $a_{i}^{\prime \prime}=\varrho a_{i}^{\prime}$ for $i=1,2$,

( $(\widetilde{\mathrm{C}} 2) b_{1,1}^{\prime}>0, \delta^{\prime}=0, b_{1,1}^{\prime \prime}>0, \delta^{\prime \prime}=0$, and there exists $\varrho>0$ such that $b_{j, k}^{\prime \prime}=\varrho b_{j, k}^{\prime}$ for $1 \leqslant j, k \leqslant 2$,

$(\widetilde{\mathrm{C}} 3) b_{1,1}^{\prime}>0, \delta^{\prime}>0, b_{j, k}^{\prime \prime}=0$ for $1 \leqslant j, k \leqslant 3$ with $(j, k) \neq(3,3)$ and $a_{i}^{\prime \prime}=0$ for $i=1,2$,

$(\widetilde{\mathrm{C}} 4) b_{1,1}^{\prime}>0, \delta^{\prime}=0, b_{j, k}^{\prime \prime}=0$ for $1 \leqslant j, k \leqslant 3$ with $(j, k) \neq(3,3)$,

$(\widetilde{\mathrm{C}} 5) b_{1,1}^{\prime \prime}>0, \delta^{\prime \prime}>0, b_{j, k}^{\prime}=0$ for $1 \leqslant j, k \leqslant 3$ with $(j, k) \neq(3,3)$ and $a_{i}^{\prime}=0$ for $i=1,2$,

( $\widetilde{\mathrm{C}} 6) b_{1,1}^{\prime \prime}>0, \delta^{\prime \prime}=0, b_{j, k}^{\prime}=0$ for $1 \leqslant j, k \leqslant 3$ with $(j, k) \neq(3,3)$,

( $\widetilde{\mathrm{C}} 7) b_{1,1}^{\prime}=0$ and $b_{1,1}^{\prime \prime}=0$,

where $\delta^{\prime}:=\sqrt{b_{1,1}^{\prime} b_{2,2}^{\prime}-\left(b_{1,2}^{\prime}\right)^{2}}$ and $\delta^{\prime \prime}:=\sqrt{b_{1,1}^{\prime \prime} b_{2,2}^{\prime \prime}-\left(b_{1,2}^{\prime \prime}\right)^{2}}$. In cases $(\widetilde{\mathrm{C}} 1),(\widetilde{\mathrm{C}} 3),(\widetilde{\mathrm{C}} 5)$ the parameters of the convolution $\mu^{\prime} * \mu^{\prime \prime}$ are $\left(a^{\prime}+a^{\prime \prime}, B^{\prime}+B^{\prime \prime}\right)$, but in the other cases it does not necessarily hold (compare with Lemma 6.2). 
Proof. First we show necessity, i.e., if $\mu^{\prime} * \mu^{\prime \prime}$ is a Gaussian measure then one of the conditions $(\widetilde{\mathrm{C}} 1)-(\widetilde{\mathrm{C}} 7)$ holds. Let us denote the parameters of the convolution $\mu^{\prime} * \mu^{\prime \prime}$ by $(a, B)$ and we write $d_{j, k}:=d_{j, k}^{ \pm \lambda}(a, B), d_{j, k}^{\prime}:=d_{j, k}^{ \pm \lambda}\left(a^{\prime}, B^{\prime}\right)$ and $d_{j, k}^{\prime \prime}:=d_{j, k}^{ \pm \lambda}\left(a^{\prime \prime}, B^{\prime \prime}\right)$ for $1 \leqslant j, k \leqslant 3$ as in Theorem 6.1. If $b_{1,1}^{\prime}>0$ and $b_{1,1}^{\prime \prime}>0$, we can easily prove that

$$
\frac{b_{1,2}}{b_{1,1}}=\frac{b_{1,2}^{\prime}}{b_{1,1}^{\prime}}=\frac{b_{1,2}^{\prime \prime}}{b_{1,1}^{\prime \prime}}, \quad \frac{b_{2,2}}{b_{1,1}}=\frac{b_{2,2}^{\prime}}{b_{1,1}^{\prime}}=\frac{b_{2,2}^{\prime \prime}}{b_{1,1}^{\prime \prime}},
$$

and $d_{2,2}^{\prime}+d_{1,1}^{\prime \prime} \in \underset{\mathbb{R}}{\mathbb{R}}$ as in Pap [10, Theorem 7.3]. This implies that there exists $\varrho>0$ such that $b_{j, k}^{\prime \prime}=\varrho b_{j, k}^{\prime}$ for $1 \leqslant j, k \leqslant 2$, i.e., $(\widetilde{\mathrm{C}} 2)$ holds.

When $b_{1,1}^{\prime}>0, \delta^{\prime}>0$ and $b_{1,1}^{\prime \prime}>0, \delta^{\prime \prime}>0$, we show that $(\widetilde{\mathrm{C}} 1)$ holds. To derive this it is sufficient to show that $b_{1,3}^{\prime \prime}=\varrho b_{1,3}^{\prime}, b_{2,3}^{\prime \prime}=\varrho b_{2,3}^{\prime}, a_{1}^{\prime \prime}=\varrho a_{1}^{\prime}$ and $a_{2}^{\prime \prime}=\varrho a_{2}^{\prime}$. Using Theorem 6.1 we obtain

(i) $\left(d_{2,2}^{\prime}+d_{1,1}^{\prime \prime}\right)\left(\operatorname{Re} d_{1,3}^{\prime}-\operatorname{Re} d_{1,3}\right)=d_{1,2}^{\prime}\left(\operatorname{Re} d_{1,3}^{\prime \prime}+\operatorname{Re} d_{2,3}^{\prime}\right)$,

(ii) $\left(d_{2,2}^{\prime}+d_{1,1}^{\prime \prime}\right)\left(\operatorname{Re} d_{2,3}^{\prime \prime}-\operatorname{Re} d_{2,3}\right)=d_{1,2}^{\prime \prime}\left(\operatorname{Re} d_{1,3}^{\prime \prime}+\operatorname{Re} d_{2,3}^{\prime \prime}\right)$,

(iii) $\left(d_{2,2}^{\prime}+d_{1,1}^{\prime \prime}\right)\left(\operatorname{Im} d_{1,3}^{\prime}-\operatorname{Im} d_{1,3}\right)=d_{1,2}^{\prime}\left(\operatorname{Im} d_{1,3}^{\prime \prime}+\operatorname{Im} d_{2,3}^{\prime}\right)$,

(iv) $\left(d_{2,2}^{\prime}+d_{1,1}^{\prime \prime}\right)\left(\operatorname{Im} d_{2,3}^{\prime}-\operatorname{Im} d_{2,3}\right)=d_{1,2}^{\prime \prime}\left(\operatorname{Im} d_{1,3}^{\prime \prime}+\operatorname{Im} d_{2,3}^{\prime}\right)$.

Let us denote $\delta_{1}^{\prime}:=b_{1,1}^{\prime} b_{2,3}^{\prime}-b_{1,2}^{\prime} b_{1,3}^{\prime}, \delta_{1}^{\prime \prime}:=b_{1,1}^{\prime \prime} b_{2,3}^{\prime \prime}-b_{1,2}^{\prime \prime} b_{1,3}^{\prime \prime}, \delta_{2}^{\prime}:=a_{1}^{\prime} b_{1,2}^{\prime}-a_{2}^{\prime} b_{1,1}^{\prime}, \delta_{2}^{\prime \prime}:=a_{1}^{\prime \prime} b_{1,2}^{\prime \prime}-a_{2}^{\prime \prime} b_{1,1}^{\prime \prime}$. Summing up (iii) and (iv) we have

$$
\left(d_{2,2}^{\prime}+d_{1,1}^{\prime \prime}\right)\left(\operatorname{Im} d_{1,3}^{\prime}+\operatorname{Im} d_{2,3}^{\prime \prime}-\operatorname{Im} d_{1,3}-\operatorname{Im} d_{2,3}\right)=\left(d_{1,2}^{\prime}+d_{1,2}^{\prime \prime}\right)\left(\operatorname{Im} d_{1,3}^{\prime \prime}+\operatorname{Im} d_{2,3}^{\prime}\right) .
$$

Using the definition of $d_{j, k}, d_{j, k}^{\prime}, d_{j, k}^{\prime \prime}(1 \leqslant j, k \leqslant 3)$ we get

$$
\begin{aligned}
& \left(\operatorname{coth}\left(\lambda \delta^{\prime}\right)+\operatorname{coth}\left(\lambda \delta^{\prime \prime}\right)\right)\left(\frac{b_{1,3}^{\prime}}{b_{1,1}^{\prime}}+\frac{\delta_{2}^{\prime}}{\lambda b_{1,1}^{\prime} \delta^{\prime} \operatorname{coth}\left(\lambda \delta^{\prime} / 2\right)}-\frac{b_{1,3}^{\prime \prime}}{b_{1,1}^{\prime \prime}}+\frac{\delta_{2}^{\prime \prime}}{\lambda b_{1,1}^{\prime \prime} \delta^{\prime \prime} \operatorname{coth}\left(\lambda \delta^{\prime \prime} / 2\right)}-\frac{2 \delta_{2}}{\lambda b_{1,1} \delta \operatorname{coth}(\lambda \delta / 2)}\right) \\
& =-\left(\frac{1}{\sinh \left(\lambda \delta^{\prime}\right)}+\frac{1}{\sinh \left(\lambda \delta^{\prime \prime}\right)}\right)\left(\frac{b_{1,3}^{\prime \prime}}{b_{1,1}^{\prime \prime}}+\frac{\delta_{2}^{\prime \prime}}{\lambda b_{1,1}^{\prime \prime} \delta^{\prime \prime} \operatorname{coth}\left(\lambda \delta^{\prime \prime} / 2\right)}-\frac{b_{1,3}^{\prime}}{b_{1,1}^{\prime}}+\frac{\delta_{2}^{\prime}}{\lambda b_{1,1}^{\prime} \delta^{\prime} \operatorname{coth}\left(\lambda \delta^{\prime} / 2\right)}\right) .
\end{aligned}
$$

An easy calculation shows that

$$
\begin{aligned}
& \left(\frac{b_{1,3}^{\prime}}{b_{1,1}^{\prime}}-\frac{b_{1,3}^{\prime \prime}}{b_{1,1}^{\prime \prime}}\right) \lambda \sinh \left(\lambda \delta^{\prime} / 2\right) \sinh \left(\lambda \delta^{\prime \prime} / 2\right) \\
& =\left(\frac{1}{\delta^{\prime}+\delta^{\prime \prime}}\left(a_{1} \frac{b_{1,2}}{b_{1,1}}-a_{2}\right)-\frac{1}{\delta^{\prime}}\left(a_{1}^{\prime} \frac{b_{1,2}^{\prime}}{b_{1,1}^{\prime}}-a_{2}^{\prime}\right)\right) \sinh \left(\lambda \delta^{\prime} / 2\right) \cosh \left(\lambda \delta^{\prime \prime} / 2\right) \\
& \quad+\left(\frac{1}{\delta^{\prime}+\delta^{\prime \prime}}\left(a_{1} \frac{b_{1,2}}{b_{1,1}}-a_{2}\right)-\frac{1}{\delta^{\prime \prime}}\left(a_{1}^{\prime \prime} \frac{b_{1,2}^{\prime \prime}}{b_{1,1}^{\prime \prime}}-a_{2}^{\prime \prime}\right)\right) \cosh \left(\lambda \delta^{\prime} / 2\right) \sinh \left(\lambda \delta^{\prime \prime} / 2\right)
\end{aligned}
$$

for all $\lambda>0$. We show that the functions

$\lambda \sinh \left(\lambda \delta^{\prime} / 2\right) \sinh \left(\lambda \delta^{\prime \prime} / 2\right), \quad \sinh \left(\lambda \delta^{\prime} / 2\right) \cosh \left(\lambda \delta^{\prime \prime} / 2\right) \quad$ and $\quad \cosh \left(\lambda \delta^{\prime} / 2\right) \sinh \left(\lambda \delta^{\prime \prime} / 2\right)$

are linearly independent on $(0 ;+\infty)$. We have

$$
\begin{aligned}
& \lambda \sinh \left(\lambda \delta^{\prime} / 2\right) \sinh \left(\lambda \delta^{\prime \prime} / 2\right)=\lambda\left(\mathrm{e}^{\lambda\left(\delta^{\prime}+\delta^{\prime \prime}\right) / 2}-\mathrm{e}^{\lambda\left(\delta^{\prime \prime}-\delta^{\prime}\right) / 2}-\mathrm{e}^{\lambda\left(\delta^{\prime}-\delta^{\prime \prime}\right) / 2}+\mathrm{e}^{-\lambda\left(\delta^{\prime}+\delta^{\prime \prime}\right) / 2}\right) / 4, \\
& \sinh \left(\lambda \delta^{\prime} / 2\right) \cosh \left(\lambda \delta^{\prime \prime} / 2\right)=\left(\mathrm{e}^{\lambda\left(\delta^{\prime}+\delta^{\prime \prime}\right) / 2}+\mathrm{e}^{\lambda\left(\delta^{\prime}-\delta^{\prime \prime}\right) / 2}-\mathrm{e}^{\lambda\left(\delta^{\prime \prime}-\delta^{\prime}\right) / 2}-\mathrm{e}^{-\lambda\left(\delta^{\prime}+\delta^{\prime \prime}\right) / 2}\right) / 4, \\
& \cosh \left(\lambda \delta^{\prime} / 2\right) \sinh \left(\lambda \delta^{\prime \prime} / 2\right)=\left(\mathrm{e}^{\lambda\left(\delta^{\prime}+\delta^{\prime \prime}\right) / 2}-\mathrm{e}^{\lambda\left(\delta^{\prime}-\delta^{\prime \prime}\right) / 2}+\mathrm{e}^{\lambda\left(\delta^{\prime \prime}-\delta^{\prime}\right) / 2}-\mathrm{e}^{-\lambda\left(\delta^{\prime}+\delta^{\prime \prime}\right) / 2}\right) / 4 .
\end{aligned}
$$

The linear independence of these functions follows from the following fact: if $c_{1}, \ldots, c_{n}$ are pairwise different complex numbers and $Q_{1}, \ldots, Q_{n}$ are complex polynomials such that $\sum_{j=1}^{n} Q_{j}(\lambda) \mathrm{e}^{c_{j} \lambda}=0$ for all $\lambda>0$ then $Q_{1}=\cdots=Q_{n}=0$. Hence we get

$$
\frac{b_{1,3}^{\prime}}{b_{1,1}^{\prime}}-\frac{b_{1,3}^{\prime \prime}}{b_{1,1}^{\prime \prime}}=0, \quad \frac{1}{\delta^{\prime}+\delta^{\prime \prime}}\left(a_{1} \frac{b_{1,2}}{b_{1,1}}-a_{2}\right)=\frac{1}{\delta^{\prime}}\left(a_{1}^{\prime} \frac{b_{1,2}^{\prime}}{b_{1,1}^{\prime}}-a_{2}^{\prime}\right)=\frac{1}{\delta^{\prime \prime}}\left(a_{1}^{\prime \prime} \frac{b_{1,2}^{\prime \prime}}{b_{1,1}^{\prime \prime}}-a_{2}^{\prime \prime}\right) .
$$


Subtracting the equation (i) from (ii) we get

$$
\left(d_{2,2}^{\prime}+d_{1,1}^{\prime \prime}\right)\left(\operatorname{Re} d_{1,3}^{\prime}-\operatorname{Re} d_{2,3}^{\prime \prime}-\operatorname{Re} d_{1,3}+\operatorname{Re} d_{2,3}\right)=\left(d_{1,2}^{\prime}-d_{1,2}^{\prime \prime}\right)\left(\operatorname{Re} d_{1,3}^{\prime \prime}+\operatorname{Re} d_{2,3}^{\prime}\right) .
$$

Using again the definition of $d_{j, k}, d_{j, k}^{\prime}, d_{j, k}^{\prime \prime}(1 \leqslant j, k \leqslant 3)$ we obtain

$$
\begin{gathered}
\left(\operatorname{coth}\left(\lambda \delta^{\prime}\right)+\operatorname{coth}\left(\lambda \delta^{\prime \prime}\right)\right)\left(\frac{a_{1}^{\prime}}{\sqrt{\lambda} b_{1,1}^{\prime}}+\frac{a_{1}^{\prime \prime}}{\sqrt{\lambda} b_{1,1}^{\prime \prime}}-\frac{2 a_{1}}{\sqrt{\lambda} b_{1,1}}+\frac{\sqrt{\lambda} \delta_{1}^{\prime}}{b_{1,1}^{\prime} \delta^{\prime} \operatorname{coth}\left(\lambda \delta^{\prime} / 2\right)}-\frac{\sqrt{\lambda} \delta_{1}^{\prime \prime}}{b_{1,1}^{\prime \prime} \delta^{\prime \prime} \operatorname{coth}\left(\lambda \delta^{\prime \prime} / 2\right)}\right) \\
=\left(\frac{1}{\sinh \left(\lambda \delta^{\prime \prime}\right)}-\frac{1}{\sinh \left(\lambda \delta^{\prime}\right)}\right)\left(\frac{a_{1}^{\prime \prime}}{\sqrt{\lambda} b_{1,1}^{\prime \prime}}-\frac{a_{1}^{\prime}}{\sqrt{\lambda} b_{1,1}^{\prime}}+\frac{\sqrt{\lambda} \delta_{1}^{\prime}}{b_{1,1}^{\prime} \delta^{\prime} \operatorname{coth}\left(\lambda \delta^{\prime} / 2\right)}+\frac{\sqrt{\lambda} \delta_{1}^{\prime \prime}}{b_{1,1}^{\prime \prime} \delta^{\prime \prime} \operatorname{coth}\left(\lambda \delta^{\prime \prime} / 2\right)}\right) .
\end{gathered}
$$

A simple calculation shows that

$$
\begin{aligned}
& \lambda\left(1+\tanh \left(\lambda \delta^{\prime} / 2\right) \tanh \left(\lambda \delta^{\prime \prime} / 2\right)\right)\left(\frac{\delta_{1}^{\prime}}{\delta^{\prime} b_{1,1}^{\prime}}-\frac{\delta_{1}^{\prime \prime}}{\delta^{\prime \prime} b_{1,1}^{\prime \prime}}\right) \\
& \quad=\left(\operatorname{coth}\left(\lambda \delta^{\prime}\right)+\operatorname{coth}\left(\lambda \delta^{\prime \prime}\right)\right)\left(2 \frac{a_{1}}{b_{1,1}}-\frac{a_{1}^{\prime}}{b_{1,1}^{\prime}}-\frac{a_{1}^{\prime \prime}}{b_{1,1}^{\prime \prime}}\right)+\left(\frac{1}{\sinh \left(\lambda \delta^{\prime}\right)}-\frac{1}{\sinh \left(\lambda \delta^{\prime \prime}\right)}\right)\left(\frac{a_{1}^{\prime}}{b_{1,1}^{\prime}}-\frac{a_{1}^{\prime \prime}}{b_{1,1}^{\prime \prime}}\right) .
\end{aligned}
$$

It can be easily checked that the functions $\lambda\left(1+\tanh \left(\lambda \delta^{\prime} / 2\right) \tanh \left(\lambda \delta^{\prime \prime} / 2\right)\right), \operatorname{coth}\left(\lambda \delta^{\prime}\right)+\operatorname{coth}\left(\lambda \delta^{\prime \prime}\right)$ and $\left(\sinh \left(\lambda \delta^{\prime}\right)\right)^{-1}-$ $\left(\sinh \left(\lambda \delta^{\prime \prime}\right)\right)^{-1}$ are linearly independent on $(0 ;+\infty)$. Hence we have

$$
\frac{a_{1}^{\prime}}{b_{1,1}^{\prime}}-\frac{a_{1}^{\prime \prime}}{b_{1,1}^{\prime \prime}}=0, \quad 2 \frac{a_{1}}{b_{1,1}}-\frac{a_{1}^{\prime}}{b_{1,1}^{\prime}}-\frac{a_{1}^{\prime \prime}}{b_{1,1}^{\prime \prime}}=0, \quad \frac{\delta_{1}^{\prime}}{\delta^{\prime} b_{1,1}^{\prime}}=\frac{\delta_{1}^{\prime \prime}}{\delta^{\prime \prime} b_{1,1}^{\prime \prime}} .
$$

Taking into account (6.2) and (6.3), we conclude that $(\widetilde{\mathrm{C}} 1)$ holds. Using Lemma 6.2 it turns out that in this case $a=a^{\prime}+a^{\prime \prime}$ and $B=B^{\prime}+B^{\prime \prime}$.

If $b_{1,1}^{\prime}>0, \delta^{\prime}>0$ and $b_{1,1}^{\prime \prime}>0, \delta^{\prime \prime}=0$ one can show that $\mu^{\prime} * \mu^{\prime \prime}$ cannot be a Gaussian measure, as in Pap [10, Theorem 7.3].

If $b_{1,1}^{\prime}>0, \delta^{\prime}>0$, and $b_{1,1}^{\prime \prime}=0$ we show that $(\widetilde{\mathrm{C}} 3)$ holds. The symmetry and positive semi-definiteness of the matrix $B^{\prime \prime}$ imply $b_{1,2}^{\prime \prime}=b_{1,3}^{\prime \prime}=0$. Lemma 6.2 yields that $b_{1,1}=b_{1,1}^{\prime}+b_{1,1}^{\prime \prime}>0$. Hence Theorem 3.1 implies that $\left(\mu^{\prime} * \mu^{\prime \prime}\right)^{\wedge}\left(\pi_{ \pm \lambda}\right)$ is an integral operator and $\operatorname{Im}\left(d_{1,1}+d_{2,2}\right)=0$ holds. By Theorem 3.1 and Theorem 6.1 we obtain $\operatorname{Im}\left(d_{1,1}+d_{2,2}\right)=\operatorname{Im}\left(d_{1,1}^{\prime}+d_{2,2}^{\prime}+\lambda b_{2,2}^{\prime \prime}\right)=\operatorname{Im}\left(\lambda b_{2,2}^{\prime \prime}\right)$. Thus $b_{2,2}^{\prime \prime}=0$, which implies that $b_{2,3}^{\prime \prime}=0$ and $\delta=\delta^{\prime}>0$. Using again Theorem 6.1 we get

$$
\begin{aligned}
& d_{1,3}=d_{1,3}^{\prime}-\sqrt{\lambda} a_{1}^{\prime \prime} d_{1,2}^{\prime}, \\
& d_{2,3}=d_{2,3}^{\prime}-\sqrt{\lambda} a_{1}^{\prime \prime} d_{2,2}^{\prime} \mp \mathrm{i} \sqrt{\lambda} a_{2}^{\prime \prime} .
\end{aligned}
$$

Taking the real part of the difference of Eqs. (6.4) and (6.5) we have

$$
2\left(\frac{a_{1}}{b_{1,1}}-\frac{a_{1}^{\prime}}{b_{1,1}^{\prime}}\right)=\lambda \delta^{\prime} \frac{a_{1}^{\prime \prime}}{b_{1,1}^{\prime}}\left(\frac{1+\cosh \left(\lambda \delta^{\prime}\right)}{\sinh \left(\lambda \delta^{\prime}\right)}\right) .
$$

Since (6.6) is valid for all $\lambda>0$, we have $a_{1}^{\prime \prime}=0$. Taking the imaginary part of (6.5) and using the fact that $a_{1}^{\prime \prime}=0$ we get

$$
a_{2}^{\prime \prime}\left(1-\frac{1}{\lambda \delta^{\prime} \operatorname{coth}\left(\lambda \delta^{\prime} / 2\right)}\right)=\frac{b_{1,3}}{b_{1,1}}-\frac{b_{1,3}^{\prime}}{b_{1,1}^{\prime}}=0 .
$$

Since (6.7) is valid for all $\lambda>0$, we get $a_{2}^{\prime \prime}=0$, so ( $\left.\widetilde{\mathrm{C}} 3\right)$ holds. If $b_{1,1}^{\prime}>0, \delta^{\prime}=0$ and $b_{1,1}^{\prime \prime}=0$ a similar argument shows that $(\widetilde{\mathrm{C}} 4)$ holds.

The aim of the following discussion is to show the converse. Suppose that $(\widetilde{\mathrm{C}} 1)$ holds. We prove that the convolution $\mu^{\prime} * \mu^{\prime \prime}$ is a Gaussian measure on $\mathbb{H}$ with parameters $\left(a^{\prime}+a^{\prime \prime}, B^{\prime}+B^{\prime \prime}\right)$. By Theorem 6.1, the Fourier transform $\left(\mu^{\prime} * \mu^{\prime \prime}\right)^{\wedge}\left(\chi_{\alpha, \beta}\right)$ equals the Fourier transform of a Gaussian measure with parameters $\left(a^{\prime}+a^{\prime \prime}, B^{\prime}+B^{\prime \prime}\right)$ at the representation $\chi_{\alpha, \beta}$ for all $\alpha, \beta>0$. Since $b_{1,1}^{\prime}+b_{1,1}^{\prime \prime}>0$, the Fourier transform $\left(\mu^{\prime} * \mu^{\prime \prime}\right)^{\wedge}\left(\pi_{ \pm \lambda}\right)$ is an integral operator on 
$L^{2}(\mathbb{R})$ with kernel function $K_{ \pm \lambda}$ given in Theorem 6.1 for all $\lambda>0$. All we have to show is that $C=C_{ \pm \lambda}\left(B^{\prime}+B^{\prime \prime}\right)$ and $V=D_{ \pm \lambda}\left(a^{\prime}+a^{\prime \prime}, B^{\prime}+B^{\prime \prime}\right)=\left(d_{j, k}^{ \pm \lambda}\left(a^{\prime}+a^{\prime \prime}, B^{\prime}+B^{\prime \prime}\right)\right)_{1 \leqslant j, k \leqslant 3}$. We have

$$
d_{2,2}^{\prime}+d_{1,1}^{\prime \prime}=\frac{\delta^{\prime} \sinh \left(\lambda(1+\varrho) \delta^{\prime}\right)}{b_{1,1}^{\prime} \sinh \left(\lambda \delta^{\prime}\right) \sinh \left(\lambda \varrho \delta^{\prime}\right)},
$$

hence using Theorem 6.1 we obtain

$$
C=\sqrt{\frac{\delta^{\prime}}{2 \pi b_{1,1}^{\prime} \sinh \left(\lambda(1+\varrho) \delta^{\prime}\right)}}=C_{ \pm \lambda}\left(B^{\prime}+B^{\prime \prime}\right) .
$$

Let $\left(\mu_{t}\right)_{t \geqslant 0}$ be a Gaussian semigroup such that $\mu_{1}$ is a Gaussian measure with parameters $\left(a^{\prime}, B^{\prime}\right)$. By the help of the semigroup property we have $\mu_{1} * \mu_{\varrho}=\mu_{1+\varrho}$. Taking into account that $a_{3}^{\prime}$ and $b_{3,3}^{\prime}$ appear only in $d_{3,3}^{ \pm \lambda}\left(a^{\prime}, B^{\prime}\right)$ (see Theorem 3.1) and the fact that $\mu_{t}$ is a Gaussian measure with parameters $\left(t a^{\prime}, t \boldsymbol{B}^{\prime}\right)$ for all $t \geqslant 0$, Theorem 3.1 and Theorem 6.1 give us

$$
v_{j, k}=d_{j, k}^{ \pm \lambda}\left(a^{\prime}+a^{\prime \prime}, B^{\prime}+B^{\prime \prime}\right)
$$

for $1 \leqslant j, k \leqslant 3$ with $(j, k) \neq(3,3)$. So we have to check only that $v_{3,3}=d_{3,3}^{ \pm \lambda}\left(a^{\prime}+a^{\prime \prime}, B^{\prime}+B^{\prime \prime}\right)$. By the help of Theorem 6.1 we get

$$
v_{3,3}=d_{3,3}^{\prime}+d_{3,3}^{\prime \prime}-\frac{1}{d_{2,2}^{\prime}+d_{1,1}^{\prime \prime}}\left(d_{3,2}^{\prime}+d_{3,1}^{\prime \prime}\right)^{2} .
$$

Calculating the real and imaginary part of (6.8) one can easily check that $v_{3,3}=d_{3,3}^{ \pm \lambda}\left(a^{\prime}+a^{\prime \prime}, B^{\prime}+B^{\prime \prime}\right)$ is valid.

Now suppose that $(\widetilde{\mathrm{C}} 2)$ holds. Using the parameters of $\mu^{\prime}$ and $\mu^{\prime \prime}$, define a vector $a=\left(a_{i}\right)_{1 \leqslant i \leqslant 3}$ and a matrix $B=\left(b_{i, j}\right)_{1 \leqslant i, j \leqslant 3}$, as in Lemma 6.2. We show that the convolution $\mu:=\mu^{\prime} * \mu^{\prime \prime}$ is a Gaussian measure on $\mathbb{H}$ with parameters $(a, B)$. An easy calculation shows that the Fourier transforms of $\mu^{\prime} * \mu^{\prime \prime}$ and $\mu$ at the one-dimensional representations coincide. Concerning the Fourier transforms at the Schrödinger representations, as in case of $(\widetilde{\mathrm{C}} 1)$, all we have to prove is that

$$
C_{ \pm \lambda}(B)=C_{ \pm \lambda}\left(B^{\prime}\right) C_{ \pm \lambda}\left(B^{\prime \prime}\right) \sqrt{\frac{2 \pi}{d_{2,2}^{\prime}+d_{1,1}^{\prime \prime}}}
$$

and $V=D_{ \pm \lambda}\left(a^{\prime}+a^{\prime \prime}, B^{\prime}+B^{\prime \prime}\right)$. Using Theorem 3.1 we have

$$
\frac{1}{\sqrt{2 \pi \lambda b_{1,1}^{\prime}}} \frac{1}{\sqrt{2 \pi \lambda b_{1,1}^{\prime \prime}}} \sqrt{\frac{2 \pi}{1 /\left(\lambda b_{1,1}^{\prime}\right)+1 /\left(\lambda b_{1,1}^{\prime \prime}\right) \pm \mathrm{i}\left(b_{1,2}^{\prime \prime} / b_{1,1}^{\prime \prime}-b_{1,2}^{\prime} / b_{1,1}^{\prime}\right)}}=\frac{1}{\sqrt{2 \pi \lambda\left(b_{1,1}^{\prime}+b_{1,1}^{\prime \prime}\right)}}=\frac{1}{\sqrt{2 \pi \lambda b_{1,1}}},
$$

since $b_{1,2}^{\prime \prime} / b_{1,1}^{\prime \prime}=b_{1,2}^{\prime} / b_{1,1}^{\prime}=\varrho$. Using similar arguments one can also easily check that $V=D_{ \pm \lambda}\left(a^{\prime}+a^{\prime \prime}, B^{\prime}+B^{\prime \prime}\right)$ holds. We note that in this case the parameters of $\mu^{\prime} * \mu^{\prime \prime}$ is not the sum of the parameters of $\mu^{\prime}$ and $\mu^{\prime \prime}$.

Suppose that $(\widetilde{\mathrm{C}} 3)$ holds. Proposition 6.1 gives us that the convolution $\mu^{\prime} * \mu^{\prime \prime}$ is a Gaussian measure on $\mathbb{H}$ with parameters $\left(a^{\prime}+a^{\prime \prime}, B^{\prime}+B^{\prime \prime}\right)$. In cases $(\widetilde{\widetilde{C}} 4),(\widetilde{\mathrm{C}} 5),(\widetilde{\mathrm{C}} 6),(\widetilde{\mathrm{C}} 7)$ we can argue as in cases $(\widetilde{\mathrm{C}} 2),(\widetilde{\mathrm{C}} 3)$. Consequently, the proof is complete.

For the proof of Theorem 1.1 we need the following lemma about the support of a Gaussian measure on $\mathbb{H}$.

Lemma 6.3. Let $\mu$ be a Gaussian measure on $\mathbb{H}$ with parameters $(a, B)$ such that $b_{1,1} b_{2,2}-b_{1,2}^{2}=0$. Let $Y_{0} \in \mathcal{H}$ be defined as in Section 2. If $\operatorname{rank}(B)=2$ then $\operatorname{supp}(\mu)=\exp \left(Y_{0}+\mathbb{R} \cdot U+\mathbb{R} \cdot X_{3}\right)$, where

$$
U:= \begin{cases}b_{1,1} X_{1}+b_{2,1} X_{2} & \text { if } b_{1,1}>0 \\ b_{2,2} X_{2} & \text { if } b_{1,1}=0 \text { and } b_{2,2}>0 .\end{cases}
$$

If $\operatorname{rank}(B)=1$ then $\operatorname{supp}(\mu)=\exp \left(Y_{0}+\mathbb{R} \cdot U+\mathbb{R} \cdot\left[Y_{0}, U\right]\right)$, where

$$
U:= \begin{cases}b_{1,1} X_{1}+b_{2,1} X_{2}+b_{3,1} X_{3} & \text { if } b_{1,1}>0, \\ b_{2,2} X_{2}+b_{3,2} X_{3} & \text { if } b_{1,1}=0 \text { and } b_{2,2}>0, \\ b_{3,3} X_{3} & \text { if } b_{1,1}=b_{2,2}=0 \text { and } b_{3,3}>0 .\end{cases}
$$

If $\operatorname{rank}(B)=0$ then $\operatorname{supp}(\mu)=\exp \left(Y_{0}\right)$. 
Proof. We apply (iii)-(v) of Lemma 4.2, respectively. If $\operatorname{rank}(B)=2$ then one can check that $\mathcal{L}\left(Y_{1}, Y_{2}\right)=\mathcal{L}\left(U, X_{3}\right)$. If $\operatorname{rank}(B)=1$ then $\mathcal{L}\left(Y_{1}\right)=\mathcal{L}(U)$.

Proof of Theorem 1.1. First we prove that if one of the conditions $(\mathrm{C} 1)$ and $(\mathrm{C} 2)$ holds then one of the conditions $(\widetilde{\mathrm{C}} 1)-(\widetilde{\mathrm{C}} 7)$ in Theorem 6.2 is valid, which implies that the convolution $\mu^{\prime} * \mu^{\prime \prime}$ is a Gaussian measure on $\mathbb{H}$.

Suppose that (C1) holds. Lemma 4.2 implies $\delta^{\prime}=\delta^{\prime \prime}=0$.

If $b_{1,1}^{\prime}=b_{1,1}^{\prime \prime}=0$ then $(\widetilde{\mathrm{C}} 7)$ holds.

If $b_{1,1}^{\prime}>0, \delta^{\prime}=0$ and $b_{1,1}^{\prime \prime}=0, \delta^{\prime \prime}=0$ we show that $(\widetilde{\mathrm{C}} 4)$ holds. It is sufficient to show that $b_{2,2}^{\prime \prime}=0$. Suppose that, on the contrary, $b_{2,2}^{\prime \prime} \neq 0$. When $\operatorname{rank}\left(B^{\prime}\right)=\operatorname{rank}\left(B^{\prime \prime}\right)=2$, by the help of Lemma 6.3, we get

$$
\operatorname{supp}\left(\mu^{\prime}\right)=\exp \left(Y_{0}^{\prime}+\mathbb{R} \cdot U^{\prime}+\mathbb{R} \cdot X_{3}\right), \quad \operatorname{supp}\left(\mu^{\prime \prime}\right)=\exp \left(Y_{0}^{\prime \prime}+\mathbb{R} \cdot U^{\prime \prime}+\mathbb{R} \cdot X_{3}\right),
$$

where $U^{\prime}=b_{1,1}^{\prime} X_{1}+b_{2,1}^{\prime} X_{2}$ and $U^{\prime \prime}=b_{2,2}^{\prime \prime} X_{2}$. Since in this case $\operatorname{supp}\left(\mu^{\prime}\right)$ and $\operatorname{supp}\left(\mu^{\prime \prime}\right)$ are contained in "Euclidean cosets" of the same 2-dimensional Abelian subgroup of $\mathbb{H}$, we obtain that $\mathcal{L}\left(U^{\prime}, X_{3}\right)=\mathcal{L}\left(U^{\prime \prime}, X_{3}\right)$. From this we conclude $b_{1,1}^{\prime}=0$, which leads to a contradiction. When $\operatorname{rank}\left(B^{\prime}\right)=1, \operatorname{rank}\left(B^{\prime \prime}\right)=2$ and in other cases one can argue similarly, so $(\widetilde{\mathrm{C}} 4)$ holds.

If $b_{1,1}^{\prime}=0, \delta^{\prime}=0$ and $b_{1,1}^{\prime \prime}>0, \delta^{\prime \prime}=0$ the same argument shows that $(\widetilde{\mathrm{C}} 6)$ holds.

If $b_{1,1}^{\prime}>0, \delta^{\prime}=0$ and $b_{1,1}^{\prime \prime}>0, \delta^{\prime \prime}=0$ we show that $(\widetilde{\mathrm{C}} 2)$ holds. When $\operatorname{rank}\left(B^{\prime}\right)=\operatorname{rank}\left(B^{\prime \prime}\right)=2, \operatorname{Lemma} 6.3$ implies that

$$
\operatorname{supp}\left(\mu^{\prime}\right)=\exp \left(Y_{0}^{\prime}+\mathbb{R} \cdot U^{\prime}+\mathbb{R} \cdot X_{3}\right), \quad \operatorname{supp}\left(\mu^{\prime \prime}\right)=\exp \left(Y_{0}^{\prime \prime}+\mathbb{R} \cdot U^{\prime \prime}+\mathbb{R} \cdot X_{3}\right),
$$

where $U^{\prime}=b_{1,1}^{\prime} X_{1}+b_{2,1}^{\prime} X_{2}$ and $U^{\prime \prime}=b_{1,1}^{\prime \prime} X_{1}+b_{2,1}^{\prime \prime} X_{2}$. Condition $(\mathrm{C} 1)$ yields that $\mathcal{L}\left(U^{\prime}, X_{3}\right)=\mathcal{L}\left(U^{\prime \prime}, X_{3}\right)$, hence we have $b_{2,1}^{\prime \prime} b_{1,1}^{\prime}=b_{2,1}^{\prime} b_{1,1}^{\prime \prime}$. Since $\delta^{\prime}=\delta^{\prime \prime}=0$ we get $b_{2,2}^{\prime \prime} b_{1,1}^{\prime}=b_{2,2}^{\prime} b_{1,1}^{\prime \prime}$. Thus ( $(\widetilde{C} 2)$ holds with $\varrho:=b_{1,1}^{\prime \prime} / b_{1,1}^{\prime}$. When $\operatorname{rank}\left(B^{\prime}\right)=\operatorname{rank}\left(B^{\prime \prime}\right)=1$, Lemma 6.3 implies that

$$
\operatorname{supp}\left(\mu^{\prime}\right)=\exp \left(Y_{0}^{\prime}+\mathbb{R} \cdot U^{\prime}+\mathbb{R} \cdot\left[Y_{0}^{\prime}, U^{\prime}\right]\right), \quad \operatorname{supp}\left(\mu^{\prime \prime}\right)=\exp \left(Y_{0}^{\prime \prime}+\mathbb{R} \cdot U^{\prime \prime}+\mathbb{R} \cdot\left[Y_{0}^{\prime \prime}, U^{\prime \prime}\right]\right),
$$

where $U^{\prime}=b_{1,1}^{\prime} X_{1}+b_{2,1}^{\prime} X_{2}+b_{3,1}^{\prime} X_{3}$ and $U^{\prime \prime}=b_{1,1}^{\prime \prime} X_{1}+b_{2,1}^{\prime \prime} X_{2}+b_{3,1}^{\prime \prime} X_{3}$. Condition (C1) yields $\mathcal{L}\left(U^{\prime},\left[Y_{0}^{\prime}, U^{\prime}\right]\right)=$

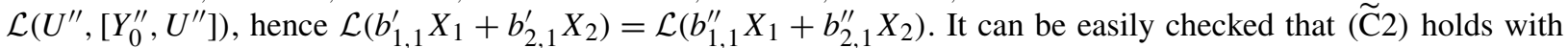
$\varrho:=b_{1,1}^{\prime \prime} / b_{1,1}^{\prime}$. When $\operatorname{rank}\left(B^{\prime}\right)=1, \operatorname{rank}\left(B^{\prime \prime}\right)=2 \operatorname{or} \operatorname{rank}\left(B^{\prime}\right)=2, \operatorname{rank}\left(B^{\prime \prime}\right)=1$ we also have $(\widetilde{\mathrm{C}} 2)$ holds.

Suppose that (C2) holds (i.e., $\mu^{\prime}=\mu_{t^{\prime}}, \mu^{\prime \prime}=\mu_{t^{\prime \prime}} * v$ or $\mu^{\prime}=\mu_{t^{\prime}} * v, \mu^{\prime \prime}=\mu_{t^{\prime \prime}}$ with appropriate nonnegative real numbers $t^{\prime}, t^{\prime \prime}$ and a Gaussian measure $v$ with support contained in the center of $\mathbb{H}$ ). Then we have

$$
\mu^{\prime} * \mu^{\prime \prime}=\mu_{t^{\prime}} * \mu_{t^{\prime \prime}} * v=\mu_{t^{\prime}+t^{\prime \prime}} * v \quad \text { or } \quad \mu^{\prime} * \mu^{\prime \prime}=\mu_{t^{\prime}} * v * \mu_{t^{\prime \prime}}=\mu_{t^{\prime}+t^{\prime \prime}} * v .
$$

Remark 6.1 and Proposition 6.1 yield that $\mu^{\prime} * \mu^{\prime \prime}$ is a Gaussian measure on $\mathbb{H}$.

Conversely, suppose that $\mu^{\prime} * \mu^{\prime \prime}$ is a Gaussian measure on $\mathbb{H}$. Then by Theorem 6.2 , one of the conditions $(\widetilde{\mathrm{C}} 1)-$ $(\widetilde{\mathrm{C}} 7)$ holds. We show that then one of the conditions $(\mathrm{C} 1)$ and $(\mathrm{C} 2)$ is valid.

Suppose that $(\widetilde{\mathrm{C}} 1)$ holds. If $b_{3,3}^{\prime \prime}-\varrho b_{3,3}^{\prime} \geqslant 0$ then let $\left(\alpha_{t}^{\prime}\right)_{t \geqslant 0}$ be a Gaussian semigroup such that $\alpha_{1}^{\prime}=\mu^{\prime}$ and let $v$ be a Gaussian measure on $\mathbb{H}$ with parameters $\left(a_{v}, B_{v}\right)$ such that

$$
B_{v}:=\left[\begin{array}{ccc}
0 & 0 & 0 \\
0 & 0 & 0 \\
0 & 0 & b_{3,3}^{\prime \prime}-\varrho b_{3,3}^{\prime}
\end{array}\right], \quad a_{v}:=\left[\begin{array}{c}
0 \\
0 \\
a_{3}^{\prime \prime}-\varrho a_{3}^{\prime}
\end{array}\right] .
$$

Remark 6.1 and Proposition 6.1 imply that $\mu^{\prime \prime}=\alpha_{\varrho}^{\prime} * \nu$, hence (C2) holds. If $b_{3,3}^{\prime \prime}-\varrho b_{3,3}^{\prime}<0$ then let $\left(\alpha_{t}^{\prime \prime}\right)_{t} \geqslant 0$ be a Gaussian semigroup such that $\alpha_{1}^{\prime \prime}=\mu^{\prime \prime}$ and let $v$ be a Gaussian measure on $\mathbb{H}$ with parameters $\left(a_{v}, B_{v}\right)$ such that

$$
B_{v}:=\left[\begin{array}{ccc}
0 & 0 & 0 \\
0 & 0 & 0 \\
0 & 0 & b_{3,3}^{\prime}-\varrho^{-1} b_{3,3}^{\prime \prime}
\end{array}\right], \quad a_{v}:=\left[\begin{array}{c}
0 \\
0 \\
a_{3}^{\prime}-\varrho^{-1} a_{3}^{\prime}
\end{array}\right] .
$$

Remark 6.1 and Proposition 6.1 imply that $\mu^{\prime}=\alpha_{1 / \varrho}^{\prime \prime} * v$, hence (C2) holds.

Suppose that $(\widetilde{\mathrm{C}} 2)$ holds. Lemma 6.3 implies that

$$
\operatorname{supp}\left(\mu^{\prime}\right) \subset \exp \left(Y_{0}^{\prime}+\mathbb{R} \cdot U^{\prime}+\mathbb{R} \cdot X_{3}\right), \quad \operatorname{supp}\left(\mu^{\prime \prime}\right) \subset \exp \left(Y_{0}^{\prime \prime}+\mathbb{R} \cdot U^{\prime \prime}+\mathbb{R} \cdot X_{3}\right),
$$


where $U^{\prime}=b_{1,1}^{\prime} X_{1}+b_{2,1}^{\prime} X_{2}$ and $U^{\prime \prime}=b_{1,1}^{\prime \prime} X_{1}+b_{2,1}^{\prime \prime} X_{2}$. Condition $(\widetilde{\mathrm{C}} 2)$ gives us that $\mathcal{L}\left(U^{\prime}\right)=\mathcal{L}\left(U^{\prime \prime}\right)$, hence $(\mathrm{C} 1)$ holds.

Suppose that $\left(\widetilde{\mathrm{C}} 3\right.$ ) holds. Let $\left(\alpha_{t}^{\prime}\right)_{t \geqslant 0}$ be a Gaussian semigroup such that $\alpha_{1}^{\prime}=\mu^{\prime}$ and let $v$ be a Gaussian measure with parameters $\left(a_{v}, B_{v}\right)$ such that

$$
B_{v}:=\left[\begin{array}{ccc}
0 & 0 & 0 \\
0 & 0 & 0 \\
0 & 0 & b_{3,3}^{\prime \prime}
\end{array}\right], \quad a_{v}:=\left[\begin{array}{c}
0 \\
0 \\
a_{3}^{\prime \prime}
\end{array}\right] .
$$

Then we have $\mu^{\prime \prime}=v=\alpha_{0}^{\prime} * v$, so (C2) holds.

Suppose that $(\widetilde{\mathrm{C}} 4)$ holds. By the help of Lemma 6.3, we have

$$
\operatorname{supp}\left(\mu^{\prime}\right) \subset \exp \left(Y_{0}^{\prime}+\mathbb{R} \cdot U^{\prime}+\mathbb{R} \cdot X_{3}\right), \quad \operatorname{supp}\left(\mu^{\prime \prime}\right) \subset \exp \left(Y_{0}^{\prime \prime}+\mathbb{R} \cdot U^{\prime \prime}\right),
$$

where $U^{\prime}=b_{1,1}^{\prime} X_{1}+b_{2,1}^{\prime} X_{2}$ and $U^{\prime \prime}=b_{3,3}^{\prime \prime} X_{3}$. Hence the support of $\mu^{\prime}$ is contained in $\exp \left(Y_{0}^{\prime}+\mathbb{R} \cdot U^{\prime}+\mathbb{R} \cdot X_{3}\right)$ and the support of $\mu^{\prime \prime}$ is contained in $\exp \left(Y_{0}^{\prime \prime}+\mathbb{R} \cdot U^{\prime}+\mathbb{R} \cdot X_{3}\right)$, so $(\mathrm{C} 1)$ holds. Similar arguments show that when ( $(\widetilde{\mathrm{C}} 5)$ holds then $(\mathrm{C} 2)$ is valid, and when $(\widetilde{\mathrm{C}} 6)$ holds then $(\mathrm{C} 1)$ is valid.

Suppose that $(\widetilde{\mathrm{C}} 7)$ holds. Using Lemma 6.3, we have

$$
\operatorname{supp}\left(\mu^{\prime}\right) \subset \exp \left(Y_{0}^{\prime}+\mathbb{R} \cdot U^{\prime}+\mathbb{R} \cdot X_{3}\right), \quad \operatorname{supp}\left(\mu^{\prime \prime}\right) \subset \exp \left(Y_{0}^{\prime \prime}+\mathbb{R} \cdot U^{\prime \prime}+\mathbb{R} \cdot X_{3}\right),
$$

where $U^{\prime}=b_{2,2}^{\prime} X_{2}$ and $U^{\prime \prime}=b_{2,2}^{\prime \prime} X_{2}$, so (C1) holds.

Remark 6.2. In case of (C1) in Theorem 1.1, $\mu^{\prime}$ and $\mu^{\prime \prime}$ are Gaussian measures also in the "Euclidean sense" (i.e., considering them as measures on $\mathbb{R}^{3}$ ), but the parameters of the convolution $\mu^{\prime} * \mu^{\prime \prime}$ is not necessarily the sum of the parameters of $\mu^{\prime}$ and $\mu^{\prime \prime}$. In case of (C2) in Theorem 1.1, $\mu^{\prime}$ and $\mu^{\prime \prime}$ are not necessarily Gaussian measures in the "Euclidean sense", but the parameters of the convolution $\mu^{\prime} * \mu^{\prime \prime}$ is the sum of the parameters of $\mu^{\prime}$ and $\mu^{\prime \prime}$.

Remark 6.3. We formulate Theorem 1.1 in the important special case of centered Gaussian measures for which the corresponding Gaussian semigroups are stable in the sense of Hazod. First we recall that a probability measure $\mu$ on $\mathbb{H}$ is called centered if

$$
\int_{\mathbb{H}} x_{1} \mu(\mathrm{d} x)=\int_{\mathbb{H}} x_{2} \mu(\mathrm{d} x)=0
$$

A convolution semigroup $\left(\mu_{t}\right)_{t \geqslant 0}$ on $\mathbb{H}$ is called centered if $\mu_{t}$ is centered for all $t \geqslant 0$. For each $t \geqslant 0$ let $d_{t}$ denote the dilation

$$
d_{t}(x)=\left(t x_{1}, t x_{2}, t^{2} x_{3}\right), \quad x \in \mathbb{H}, t \geqslant 0 .
$$

By Hazod [6, page 229], a Gaussian semigroup $\left(\mu_{t}\right)_{t \geqslant 0}$ is centered and stable in the sense that $\mu_{t}=d_{\sqrt{t}} \mu_{1}, t \geqslant 0$ (Hazod stability) if and only if its infinitesimal generator has the form

$$
a_{3} X_{3}+\frac{1}{2} \sum_{i=1}^{2} \sum_{j=1}^{2} b_{i, j} X_{i} X_{j}
$$

Wehn [17] proved the following central limit theorem. Let $|\cdot|$ be a fixed homogeneous norm on $\mathbb{H}$ and let us consider a centered probability measure $\mu$ on $\mathbb{H}$. If $\int_{\mathbb{H}}|x|^{2} \mu(\mathrm{d} x)<+\infty$, then $\left(d_{1 / \sqrt{n}}\left(\mu^{* n}\right)\right)_{n} \geqslant 1$ converges towards $v$ weakly, where $v$ is a Gaussian measure on $\mathbb{H}$ such that the corresponding Gaussian semigroup has infinitesimal generator (6.9).

For centered and stable Gaussian measures Theorem 1.1 has the following form.

Let $\mu^{\prime}$ and $\mu^{\prime \prime}$ be Gaussian measures on $\mathbb{H}$ such that the corresponding Gaussian semigroups have infinitesimal generators

$$
a_{3}^{\prime} X_{3}+\frac{1}{2} \sum_{i=1}^{2} \sum_{j=1}^{2} b_{i, j}^{\prime} X_{i} X_{j} \quad \text { and } \quad a_{3}^{\prime \prime} X_{3}+\frac{1}{2} \sum_{i=1}^{2} \sum_{j=1}^{2} b_{i, j}^{\prime \prime} X_{i} X_{j}, \quad \text { respectively. }
$$

Then the convolution $\mu^{\prime} * \mu^{\prime \prime}$ is a Gaussian measure on $\mathbb{H}$ if and only if there exist $t^{\prime}, t^{\prime \prime} \geqslant 0$, a Gaussian semigroup $\left(\mu_{t}\right)_{t \geqslant 0}$ with infinitesimal generator (6.9) and an element $x \in \mathbb{H}$ which is contained in the center of $\mathbb{H}$ such that either 
$\mu^{\prime}=\mu_{t^{\prime}}, \mu^{\prime \prime}=\mu_{t^{\prime \prime}} * \varepsilon_{x}$ or $\mu^{\prime}=\mu_{t^{\prime}} * \varepsilon_{x}, \mu^{\prime \prime}=\mu_{t^{\prime \prime}}$ holds. Moreover, in this case $a_{3}=a_{3}^{\prime}+a_{3}^{\prime \prime}$ and $b_{i, j}=b_{i, j}^{\prime}+b_{i, j}^{\prime \prime}$, $1 \leqslant i, j \leqslant 2$.

The proof of this statement can be carried out in a direct way applying Theorem 7.3 in Pap [10], and Lemma 6.2 and Proposition 6.1 of the present paper.

\section{Acknowledgements}

The authors are very grateful to the unknown referees for their important observations and remarks that helped to improve the presentation of the paper.

The authors have been supported by the Hungarian Scientific Research Fund under Grant No. OTKA-T048544/ 2005. The first author has been also supported by the Hungarian Scientific Research Fund under Grant No. OTKAF046061/2004.

\section{References}

[1] R.B. Ash, Real Analysis and Probability, Academic Press, New York, 1972.

[2] P. Baldi, Unicité du plongement d'une mesure de probabilité dans un semi-groupe de convolution gaussien, Cas non-abélien, Math. Z. 188 (1985) 411-417.

[3] M. Chaleyat-Maurel, Densités des diffusions invariantes sur certains groupes nilpotents. Calcul d'aprés B. Gaveau, Astérisque 84-85 (1981) 203-214.

[4] E.B. Davies, Heat Kernels and Spectral Theory, Cambridge University Press, 1989.

[5] I.S. Gradshteyn, I.M. Ryzhik, Table of Integrals, Series, and Products, Academic Press, New York, 1965.

[6] W. Hazod, E. Siebert, Stable Probability Measures on Euclidean Spaces and on Locally Compact Groups, Kluwer Academic Publishers, Drodrecht, 2001.

[7] H. Heyer, Probability Measures on Locally Compact Groups, Springer, Berlin, 1977.

[8] D. Neuenschwander, Probabilities on the Heisenberg group: Limit theorems and Brownian motion, in: Lecture Notes in Math., vol. 1630, Springer, Berlin, 1996, pp. 379-397.

[9] G. Pap, Uniqueness of embedding into a Gaussian semigroup on a nilpotent Lie group, Arch. Math. (Basel) 62 (1994) $282-288$.

[10] G. Pap, Fourier transform of symmetric Gauss measures on the Heisenberg group, Semigroup Forum 64 (2002) $130-158$.

[11] K.R. Parthatsarathy, Probability Measures on Metric Spaces, Academic Press, New York, 1967.

[12] B. Roynette, Croissance et mouvements browniens d'un groupe de Lie nilpotent et simplement connexe, Z. Wahr. Verw. Gebiete 32 (1975) 133-138.

[13] E. Siebert, Fourier analysis and limit theorems for convolution semigroups on a locally compact group, Adv. Math. 39 (1981) $111-154$.

[14] E. Siebert, Absolute continuity, singularity, and supports of Gauss semigroups on a Lie group, Monatsh. Math. 93 (1982) $239-253$.

[15] M.E. Taylor, Noncommutative Harmonic Analysis, Math. Surveys Monogr., vol. 22, American Mathematical Society, Providence, RI, 1986.

[16] W. Tomé, The Representation Independent Propagator for General Lie Groups, World Scientific, Singapore, 1998.

[17] D. Wehn, Probabilities on Lie groups, Proc. Natl. Acad. Sci. USA 48 (1962) 791-795. 